Article

\title{
SMM-ET: An SMM Evaluation Tool for the Quantitative Treatment of Ac Susceptibility and Magnetic Hysteresis Data
}

\author{
Athanassios K. Boudalis \\ Institut de Chimie de Strasbourg (UMR 7177, CNRS-Unistra), Université de \\ Strasbourg, 4 rue Blaise Pascal, CS 90032, F-67081 Strasbourg, France; \\ Email: abountalis@unistra.fr; Tel.: +33-(0)-3-6885-5350
}

\begin{abstract}
Ac susceptometry and magnetic hysteresis studies are the two most used techniques for the basic characterization of magnetic relaxation properties of Single-Molecule Magnets. Nevertheless, the full quantitative treatment of such studies is rarely carried out, in particular as regards the absolute magnitudes of the in-phase $\left(\chi^{\prime}\right)$ and out-of-phase $\left(\chi^{\prime \prime}\right)$ ac susceptibility signals, and the exact shapes of hysteresis loops. To facilitate such quantitative analyses, an SMM evaluator tool has been developed. It uses the dc magnetic susceptibility/magnetization properties of any SMM, and the parameters characteristic of the various relevant relaxation processes (Orbach, Raman, Direct, QTM) to calculate the exact ac susceptibility/magnetic hysteresis curves under any temperature, magnetic field and ac frequency or dc field scan rate. It also implements a model that calculates the actual fraction of molecules that contribute to the SMM effect, as well as models which account for distributions of the relaxation times. Indicative examples of a "strong", a "medium" and a "weak" SMM are analysed with this tool, demonstrating the additional information that can be extracted by quantitative treatment of such data.
\end{abstract}

KEYWORDS: Single-Molecule Magnets; ac susceptibility; magnetic hysteresis; modeling

\section{G Open Access}

Received: 28 November 2019

Accepted: 18 March 2020

Published: 28 April 2020

Copyright (C) 2020 by the author(s). Licensee Hapres, London, United Kingdom. This is an open access article distributed under the terms and conditions of Creative Commons Attribution 4.0 International License.

\section{ABBREVIATIONS}

SMM, Single-Molecule Magnets; ac, alternating current; dc, direct current; QTM, Quantum Tunneling of the Magnetization; FC-ZFC, field-cooled zero-field-cooled

\section{INTRODUCTION}

It would be probably fair to say that from the moment Single-Molecule Magnets (SMMs) entered the field of molecular magnetism almost three decades ago, ac susceptometry has been the workhorse of this research, starting from its foundational paper [1]. Today, ac susceptometry is readily accessible from commercial magnetometers, it allows the 
determination of kinetic parameters of the magnetic relaxation processes as a function of temperature and static magnetic fields and it can yield kinetic information much more conveniently that isothermal magnetic field sweeps or FC-ZFC studies, particularly in the case of fast processes.

The most easily accessible piece of information derived from ac susceptometry is the out-of-phase magnetization $\left(\chi^{\prime \prime}\right)$, which forms peaks whose positions are characteristic of the magnetic relaxation time. Peak maxima in the isothermal $\chi^{\prime \prime}=f(\omega)$ representation, or in the isofrequency $\chi^{\prime \prime}=f(T)$ representation, are characteristic of the relaxation time at that particular temperature or frequency, respectively. Collecting several peak positions from isothermal $\chi^{\prime \prime}=f(\omega)$ experiments conducted at different temperatures (or isofrequency $\chi^{\prime \prime}=f(T)$ experiments conducted at different frequencies), allows us to model the characteristic relaxation times to account for a series of relaxation mechanisms.

The easy accessibility to this information, and the high attention that is given to peak positions, obscures another important parameter, i.e., their intensities. Moreover, as the appearance this out-of-phase component is associated with the decrease of the in-phase component, a quantitative assessment of the latter is also relevant, but not routinely carried out.

At the same time, magnetic field sweeps are the second most diagnostic experiment, also used from the earliest studies in the field [2]. These reveal hysteresis loops when the relaxation is sufficiently slow, despite the fact that the timescales of this experiment are much longer than the ac experiment. Such experiments are also critical in determining magnetic field positions at which magnetic level crossings induce accelerated relaxation through Quantum Tunneling of the Magnetization (QTM). However, apart from the descriptive treatment of these experiments (remanent magnetization, coercive field, QTM-induced steps), quantitative analyses of experimental data are extremely rare [3-6]. Partly responsible for this paucity of analyses is the lack of analytical descriptions of these curves, whose reproduction requires the numerical solution of the associated differential equation. While the respective differential equations describing the ac experiment were analytically solved several decades ago, this is not the case with magnetic field sweep experiments.

Herein, it will be shown that additional information on the relaxation processes of SMMs can be derived from the quantitative treatment of: (i) the magnitudes of the $\chi^{\prime}$ and $\chi^{\prime \prime}$ ac signals and (ii) the shapes of hysteresis loops. This additional information does not only concern the kinetic parameters of the relaxation processes of unique molecules, but also the determination of the slowly relaxing fraction, i.e., the amount of molecules in the sample that do relax at the determined finite rates. Such subtle distinctions are becoming more important, as we are beginning to understand that the SMM phenomenon is not uniquely determined by the properties of the single molecule, as the name implies, but also by those of its surroundings [7,8]. 
To facilitate such analyses an SMM evaluation tool has been developed and its functionalities will be presented through the analysis of SMMs previously reported in the literature. This analysis will also extend to the description of distribution mechanisms that are employed to interpret non-ideal relaxation processes, and it will compare the validity of such distributions with that of low fractions of slowly relaxing molecules.

\section{BASIC THEORY OF DYNAMIC MAGNETIZATION EXPERIMENTS}

\section{The Ac Experiment}

During the ac susceptibility experiment, a weak (a few G) and harmonically oscillating magnetic field $H_{a c}(t)=H_{0} \sin (\omega t)$ is applied to the sample, giving rise to a complex magnetic susceptibility response. This can be analyzed to a real component that follows the magnetic field's frequency (in-phase) and to an imaginary one which lags behind (out-of-phase). A static (dc) magnetic field $H_{d c}$ may also be applied on top of the oscillating field, but this is not mandatory.

The magnetization of the sample will be time-dependent and can be written as a sum of time-independent $\mathrm{dc}$ component and a time-dependent ac component:

$$
M(t, T, H)=M_{e q, d c}\left(T, H_{d c}\right)+M_{a c}\left(T, H_{a c}(t)\right)
$$

Assuming a linear response, we can define:

$$
\begin{gathered}
\chi_{T}\left(T, H_{d c}\right)=\frac{\mathrm{d} M_{e q, d c}\left(T, H_{d c}\right)}{\mathrm{d} H_{d c}}=\frac{M_{T}\left(T, H_{d c}\right)}{H_{d c}} \\
\chi_{S}\left(T, H_{d c}, H_{0}\right)=\frac{\mathrm{d} M_{e q, a c}\left(T, H_{d c}, H_{0}\right)}{\mathrm{d} H_{a c}(t)}=\frac{M_{S}\left(T, H_{d c}, H_{0}\right)}{H_{a c}(t)}=\frac{M_{S}\left(T, H_{d c}, H_{0}\right)}{H_{0} \sin (\omega t)}
\end{gathered}
$$

Here, $\chi_{T}$ is the isothermal susceptibility, and corresponds to the static susceptibility, i.e., its equilibrium value under a static magnetic field $(\omega \rightarrow$ 0 ); experimentally, it is the value determined by a dc experiment. Moreover, $\chi_{s}$ is the adiabatic susceptibility, which follows the magnetic field without any lag and attains its equilibrium value instantaneously, it therefore corresponds to the magnetic susceptibility at the limit $\omega \rightarrow \infty$.

By replacing in the above equation it becomes:

$$
M\left(t, T, H_{d c}\right)=\chi_{T}\left(T, H_{d c}\right) H_{d c}+\chi_{s}\left(T, H_{d c}\right) H_{0} \sin (\omega t)
$$

The experiment is described by the following differential equation, where for simplicity we have replaced $H=H_{d c}$ :

$$
\tau(T, H) \frac{\mathrm{d} M(t, T, H)}{\mathrm{d} t}+M(t, T, H)=\chi_{T}(T, H) H+\chi_{S}(T, H) H_{0} \sin (\omega t)
$$

The solution to the equation has been given analytically by Casimir and du Pré, who proposed a model for spin-lattice relaxation [9].

$$
\chi^{\prime}=\chi_{S}+\frac{\chi_{T}-\chi_{S}}{1+(\omega \tau)^{2}}
$$




$$
\chi^{\prime \prime}=\left(\chi_{T}-\chi_{S}\right) \frac{\omega \tau}{1+(\omega \tau)^{2}}
$$

These relations describe the behavior of an ideal sample, with a unique relaxation time $\tau$, and whose molecules all relax slowly with that characteristic time. The basics of this theory have been explained in great detail elsewhere $[10,11]$ and will not be covered here. It is interesting to note, however, that Bloch [12] undertook a similar treatment for an oscillating magnetic field $\mathbf{H}_{1}$ transversely superposed on a static field $\mathbf{H}_{0}$, deriving relaxation times for magnetic resonance.

Assuming that all the molecules in the sample will eventually be blocked, i.e. will relax slowly, at some sufficiently large frequency it should hold that $\chi_{s}=0$ and the above relations will read:

$$
\begin{gathered}
\chi^{\prime}=\frac{\chi_{T}}{1+(\omega \tau)^{2}} \\
\chi^{\prime \prime}=\chi_{T} \frac{\omega \tau}{1+(\omega \tau)^{2}}
\end{gathered}
$$

It is easily seen that at for a frequency such that $\omega \tau=1, \chi^{\prime \prime}$ is maximized at value $\chi^{\prime \prime}=\chi_{T} / 2$ and that for that frequency $\chi^{\prime}=\chi_{T} / 2$, i.e. $\chi^{\prime \prime}=$ $\chi^{\prime}$. However, if only a fraction, $\rho$, undergoes slow magnetic relaxation the out-of-phase signal should be scaled according to that fraction, i.e.:

$$
\chi^{\prime \prime}=\rho \chi_{T} \frac{\omega \tau}{1+(\omega \tau)^{2}}
$$

We may notice a correspondence of this relation to the previous, in which:

$$
\rho \chi_{T}=\chi_{T}-\chi_{S} \Rightarrow \rho=\frac{\chi_{T}-\chi_{S}}{\chi_{T}}=1-\frac{\chi_{S}}{\chi_{T}}
$$

which indicates that the difference $\chi_{T}-\chi_{S}$ corresponds to the number of slowly relaxing molecules.

Through simple algebraic manipulation of the above relation we may rewrite the in-phase component as:

$$
\chi^{\prime}=(1-\rho) \chi_{T}+\rho \chi_{T} \frac{1}{1+(\omega \tau)^{2}}
$$

which points to a fraction $1-\rho$ which behaves like in a dc experiment, while the rest of the molecules undergo slow relaxation.

In standard presentations of the ac experiment it is pointed out that the maximum value of $\chi^{\prime \prime}$ is $\left(\chi_{T}-\chi_{S}\right) / 2$, which means that as $\chi_{S}$ approaches $\chi_{T}$ the out-of-phase signal decreases. In the extreme case of $\chi_{T}=\chi_{S}$ (hence $\rho=0$ ) the out-of-phase signal is entirely suppressed. This is in agreement with the conclusion of a zero slowly relaxing fraction. Stated this way, this description may imply for magnetochemists that $\chi_{s}$ is a property which equally characterizes each individual molecule. However, it should be noted that the above concepts derive from quantum statistical 
considerations (for a rigorous discussion see ref. [13]) and care should be taken when they are extrapolated to single molecules. Thus, it is more appropriate to consider $\chi_{s}$ as a statistical parameter, meaningful only for large ensembles. As such, it introduces a sharp distinction between slow and fast relaxing molecules, but not in the context of two different but finite relaxation rates (monodisperse or distributed). Rather, it introduces a distinction between finitely and infinitely fast relaxations.

It follows from the above discussion that an additional layer of information is hidden in the intensities of the out-of-phase signals, which are typically overlooked. This information pertains to the statistical behavior of the sample, and to the fraction of molecules which contribute to the SMM phenomenon. We had alluded to the above considerations several years ago in the context of our study of a $\mathrm{Ni}_{5}{ }_{5}$ ferromagnetic cluster exhibiting weak out-of-phase signals [14], also citing several indicative examples of SMMs with such weak signals. It may be added to those examples that members of the $\mathrm{Mn}_{12}$ family have often exhibited weaker than predicted out-of-phase signals [15-21], an observation not so easy to make when ac data are plotted as $\chi^{\prime}{ }_{M} T$ vs $T$ for the in-phase component according to prevailing practice. Relevant remarks have been made by others [22-26] though such discussions do not constitute a routine part of the analysis of ac susceptometry experiments of SMMs.

It should be stated that $\rho$ may be a function of temperature or applied magnetic field, since these parameters may activate/block different relaxation pathways. However, the precise origins of those dependencies should be quite complicated to determine, and they should vary as a function of the SMM structure, or even the sample nature. Herein, absolutely no assumptions are made as to the mechanistic details of these dependencies. These are clearly beyond the scope of this general purpose tool, which only provides a phenomenological framework to quantify their net effect by calculating $\rho$. Indeed, in the following treatment, $\rho$ will be considered as a purely phenomenological parameter, disregarding mechanistic considerations.

In closing this short presentation, a key point needs to be made, whose importance will become clear in the following discussion. Considering a $\chi^{\prime \prime}=f(\omega)$ peak, its shape will be described by the condition under which at two frequencies $\omega_{1}$ and $\omega_{2}$ it holds that $\chi^{\prime \prime}\left(\omega_{1}\right)=\chi^{\prime \prime}\left(\omega_{2}\right)$. Using the equation of the monodisperse system, it is easy to prove that this condition is fulfilled when $\omega_{1} \omega_{2} \tau=1$, which describes any pair of points on the two sides of the peak, and which are symmetric when plotted on a logarithmic $x$-axis. To calculate the linewidth as the full width at half-maximum (FWHM), we search the $\omega$ values for which $\chi^{\prime \prime}(\omega)=\chi^{\prime \prime} \max / 2$ $=\left(\chi_{T}-\chi_{S}\right) / 4$. The solution of the resulting quadratic equation yields $\omega \tau=$ $2 \pm \sqrt{3}$, for which $\Delta \omega_{\mathrm{FWHM}}=2 \sqrt{3} / \tau$, hence the peak width is only a function of $\tau$. This observation explains the apparent widening of the out-of-phase peaks upon increasing frequency (or temperature), as the concomitant decrease of $\tau(T, H)$. 
This is a key point to keep in mind, as it provides a simple yet powerful criterion in assessing deviations from a monodisperse system.

\section{The Field-Sweep Experiment}

The field-sweep experiment also involves a variable magnetic field, but in this case this is varied linearly between two opposite, and usually large, values $\pm H_{\max }$ (which may reach several T). Assuming a field scan rate $\kappa=\mathrm{d} H / \mathrm{d} t$, and an equilibrium magnetization $M_{\text {eq }}(T, H)$, then the magnetization function $M(t, T, H)$ will obey:

$$
\tau(T, H) \frac{\mathrm{d} M(t, T, H(t))}{\mathrm{d} t}+M(t, T, H(t))=M_{e q}(T, H)
$$

This may be rewritten as:

$$
\frac{\mathrm{d} M(t, T, H(t))}{\mathrm{d} t}=\frac{\mathrm{d} M(t, T, H(t))}{\mathrm{d} H} \frac{\mathrm{d} H}{\mathrm{~d} t}=-\frac{M(t, T, H(t))-M_{e q}(T, H)}{\tau(T, H)}
$$

or

$$
\frac{\mathrm{d} M(t, T, H(t))}{\mathrm{d} H}=-\frac{M(t, T, H(t))-M_{e q}(T, H)}{\kappa \tau(T, H)}
$$

This differential equation assumes a monodisperse relaxation time $\tau(T, H)$ and a sample fully undergoing slow relaxation. If, however, only a fraction $\rho$ undergoes slow magnetic relaxation, this equation becomes:

$$
\frac{\mathrm{d} M(t, T, H(t))}{\mathrm{d} H}=-\frac{M(t, T, H(t))-M_{e q}(T, H)}{\rho \kappa \tau(T, H)}
$$

This differential equation contains as parameters the equilibrium value of the magnetization $M_{e q}(T, H)$ and the relaxation time $\tau(T, H)$, both of which are functions of the magnetic field. Therefore, it needs to be solved at each different magnetic field of the field-sweep experiment using the parameter values at that specific field. Not only is an analytical solution not possible for such a problem, but the numerical solution requires specific considerations (see below).

\section{RESULTS}

\section{Modeling the Ac Susceptibility Intensities and Magnetic Hysteresis Shapes of Real Molecules}

In describing the previous theoretical background, it may be noted that $\chi^{\prime}$ and $\chi^{\prime \prime}$ are often presented as functions of the abstract susceptibilities $\chi_{T}$ and $\chi_{S}$, and of an abstract time constant $\tau$. In addition, in theoretical treatments of the ac experiment $\chi^{\prime}$ and $\chi^{\prime \prime}$ are given as unitless fractions of $\chi_{T}$, which is thus removed from the discussion. The fact that all these parameters are functions of $T$ and $H$, and that they have specific forms and magnitudes depending on the spin Hamiltonian parameters (for $\chi_{T}$ and $\chi_{S}$ ) and on the relative contributions of the various relaxation mechanisms (for $\tau$ ) is not explicitly addressed. This precludes 
the treatment of some quantitative aspects of the ac susceptometry experiment.

\section{Modeling of $\tau(T, H)$}

This is the primary function that needs to be explicitly modeled for a quantitative treatment of relaxation data at various temperatures and magnetic fields. The SMM evaluation tool considers several processes which have been found to intervene in magnetic relaxation in SMMs. In the relevant literature, a popular parametrization scheme accounts for the Orbach, Raman, direct and QTM [27,28] processes, and is given as:

$$
\tau(T, H)^{-1}=b_{\text {Orbach }} e^{-U_{\text {eff }} / k_{B} T}+b_{\text {Raman }} T^{n}+b_{\text {Direct }} H^{m} T+\frac{b_{Q T M_{1}}}{1+b_{Q T M_{2}}\left(H-H_{Q T M(i)}\right)^{2}}
$$

where $m=2$ (or 4) for non-Kramers (or Kramers) systems, $b_{\text {orbach }}$ is the inverse of the pre-exponential factor $\tau_{0}$ and $H_{Q T M(i)}$ is the level-crossing field closest to $H$. This model reproduces the effects of QTM, the most obvious of which are steps in the $M$ vs $H$ hysteresis curves, at the magnetic fields of the level crossings. These level-crossing positions are not theoretically calculated, but manually introduced at previously determined values.

A

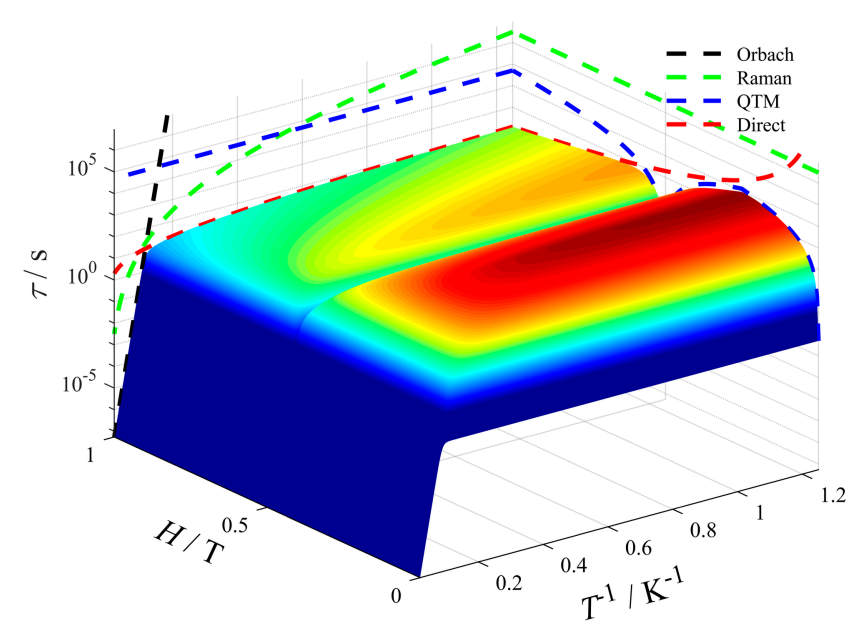

B

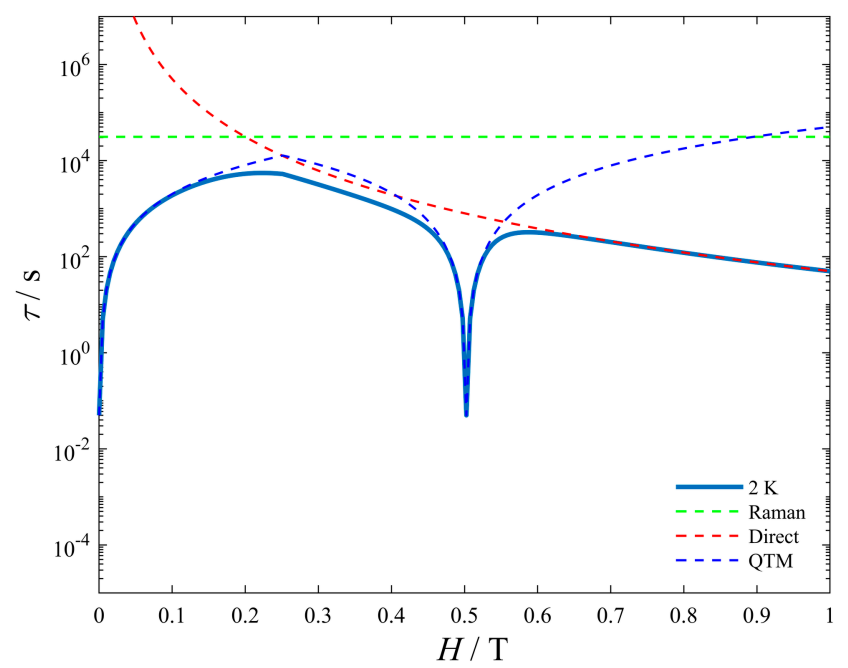

Figure 1. (A) The $\tau(T, H)$ function for an indicative set of parameters: $U_{\text {eff }}=200 \mathrm{~K}, b_{\text {Orbach }}=10^{9} \mathrm{~s}^{-1}, n=5$, $b_{\text {Raman }}=10^{-6} \mathrm{~s}^{-1} \mathrm{~K}^{-5}, m=4, b_{\text {Direct }}=10^{-2} \mathrm{~s}^{-1} \mathrm{~K}^{-1} \mathrm{~T}^{-4}, b_{\text {QTM} 1}=20 \mathrm{~s}^{-1}, b_{\text {QTM } 2}=10^{6} \mathrm{~T}^{-2}, H_{\text {QTM }}=[0,0.5 \mathrm{~T}]$. (B) The $\tau(2 \mathrm{~K}$, $H$ ) curve shows the relaxation time decrease due to QTM at the level-crossing fields of 0 and $0.5 \mathrm{~T}$.

Each of the terms can be further refined depending on the technique used and the system studied, e.g., to include the $U_{e f f}{ }^{3}$ dependence in the Orbach process [29], the Brons-van Vleck field-dependent correction in the Raman process [30], the coth dependence in the direct process, etc. However, for the purposes of the present study the above scheme is a useful basis of comparison, as it has been used to describe a large number of systems. Thus, the SMM evaluation tool takes as inputs the 
eight kinetic parameters of the above equation, plus any number of additional $H_{Q T M}$ fields. The resulting $\tau(T, H)$ function can be visualized as a surface. An indicative plot is given in Figure 1.

\section{Modeling of $\chi_{T}(T, H)$}

A good approximation of the $\chi_{T}(T, H)$ function can be obtained from dc experiments. If fits to the dc data have been possible, then the $\chi_{T}(T, H)$ function can be parametrically reproduced for any point in the $(T, H)$ space using the spin Hamiltonian parameters and tools such as Easyspin [31], Phi [32], MAGPACK [33] or other. Thus, $\chi_{T}(T, H)$ can be calculated for the temperature and magnetic field domains of the ac experiment, even if these are different from those of the dc experiment, which is usually the case.

However, fits to the dc data may not have been possible, e.g., in the case of very large Hamiltonian matrices, or in the case of molecules with strong spin-orbit couplings that preclude the use of the spin Hamiltonian approach, thus complicating analysis. In that case, $\chi_{T}$ can be calculated for the $T$ and $H$ domains of the ac experiment by interpolation of the dc experimental data. This method is more restrictive than the parametric reproduction of the function; e.g., starting from $\chi_{M}$ vs $T$ dc data, interpolations are valid, strictly speaking, for the $H$ values under which these dc data were collected, and vice vers $a$ for the temperatures of the $M$ vs $H$ experiments. On the other hand it provides a useful and tractable alternative when parametric calculations are impossible (see below), or when they are possible but computationally expensive.

\section{Modeling of relaxation time distributions}

Several models have been developed to describe samples with distributions of their relaxation times, such as proposed by Cole and Cole [34] according to the generalised Debye model, by Davidson and Cole [35], and a more generalized form by Havriliak and Negami [36]. Although these were proposed to describe dielectric susceptibilities, they have found wide application in magnetic susceptibility studies. The current implementation of the tool considers the generalized Debye model proposed by Cole and Cole [34]:

$$
\begin{aligned}
& \chi^{\prime}(\omega, T, H)=\chi_{S}+\left(\chi_{T}(T, H)-\chi_{S}\right) \frac{1+(\omega \tau(T, H))^{1-\alpha} \sin (\pi \alpha / 2)}{1+2(\omega \tau(T, H))^{1-\alpha} \sin (\pi \alpha / 2)+(\omega \tau(T, H))^{2(1-\alpha)}} \\
& \chi^{\prime \prime}(\omega, T, H)=\left(\chi_{T}(T, H)-\chi_{S}\right) \frac{(\omega \tau(T, H))^{1-\alpha} \cos (\pi \alpha / 2)}{1+2(\omega \tau(T, H))^{1-\alpha} \sin (\pi \alpha / 2)+(\omega \tau(T, H))^{2(1-\alpha)}}
\end{aligned}
$$

This distribution parametrization is very popular, especially as it allows for an analytical expression of the measurable quantities. However, the empirical parameter $\alpha$ is not directly related to any specific physical quantity or process. Thus, the more fundamental source of distributions in relaxation times is not addressed. 
To do so, the tool implements distributions of the spin reversal barrier $U_{\text {eff }}$ offering a choice between Gaussian, Lorentzian or Voigtian probability density functions. Indeed, it was shown by Weihe and coworkers [37], and subsequently by this author [38] and others [39] that the explicit consideration of distributions of fundamental spin Hamiltonian parameters is required to fully explain the shapes of Electron Paramagnetic Resonance spectra. With the $U_{\text {eff }}$ barrier being the key parameter that defines whether a molecule functions as an SMM, and with enormous efforts having been made for its maximization over the past two decades [40-48], it is equally relevant to assess how distributions of its value are manifested in ac experiments. Indeed, the magnitude of $U_{\text {eff }}$ is directly determined by structural parameters around metal ions through their effect on crystal-field or spin-Hamiltonian parameters. Since atomic positions of ligand atoms are subject to vibrational effects (quantified by the anisotropic displacement tensor elements $U_{i j}$ of the crystal structures), the resulting positional distributions should ultimately give rise to $U_{\text {eff }}$ distributions. Since the SMM effect is essentially a relaxation over the $U_{\text {eff }}$ barrier, it is critical to assess whether any distributions of that barrier have visible effects on the observed magnetic behaviours. Similar considerations are addressed by the CC-FIT2 program [49], which associates the Debye model distributions with a log-normal distribution of the relaxation times $\tau$.

For the hypothetical SMM whose $\tau(T, H)$ function is given in Figure 1, the distributions of the resulting relaxation times are shown in Figure 2 under a magnetic field of $0.1 \mathrm{~T}$. From that figure it is observed that normal distributions of $U_{\text {eff }}$ result in lognormal distributions of $\tau$, at the temperature ranges where the Orbach process is dominant. Indeed, in the plots on the left column, where $\tau$ is expressed in a linear scale, its distribution is non-symmetric, and becomes symmetric when it is plotted on a logarithmic scale (right column). Moreover, $\tau$ becomes distributed only at higher temperatures, where the Orbach process is dominant (see Figure 1A). At lower temperatures, where direct and Raman processes are dominant, $\tau$ is essentially monodisperse, as these processes are not influenced by $U_{\text {eff }}$ (and its distributions). In other words, for the same $\sigma U_{\text {eff, }}$ the width of resulting distribution of $\tau$ depends on the temperature (and magnetic field), as these parameters determine how dominant the Orbach process becomes.

It is interesting to note that while the monodisperse curve $\tau_{\text {mono }}(T)$ closely follows the maxima (modes) of the distributions, the weighted average value $\tau_{\text {avg }}(T)$ diverges at higher temperatures $\left(\tau_{\text {avg }}(T)=\right.$ $\Sigma\left[\tau\left(U_{\text {eff }}, T\right) \cdot w\left(U_{\text {eff }}\right)\right]$, where $w\left(U_{\text {eff }}\right)$ is the function of the weight distribution

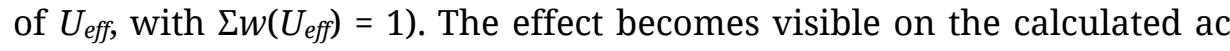
susceptibility curves (see below).

By implementing the generalized Debye model and actual $U_{\text {eff }}$ distributions on the magnetic susceptibility curves calculated for the hypothetical SMM of our example, we can calculate the ac susceptibility 
experiments at various temperatures, magnetic fields and frequencies and plot them as isothermal/isofield plots, either as $\chi^{\prime}, \chi^{\prime \prime}(f)$ or as $\chi^{\prime \prime}\left(\chi^{\prime}\right)$ (i.e., Argand or Cole-Cole plots). We can also plot them as isofrequency/isofield $\chi^{\prime}, \chi^{\prime \prime}(T)$ plots. In Figure 3 are shown all these alternative representations, for both distribution models and for two different sample types: a fully relaxing $\left(\chi_{S} / \chi_{T}=0\right)$ and one relaxing with $80 \%$ of its molecules $\left(\chi_{S} / \chi_{T}=0.2\right)$.

A

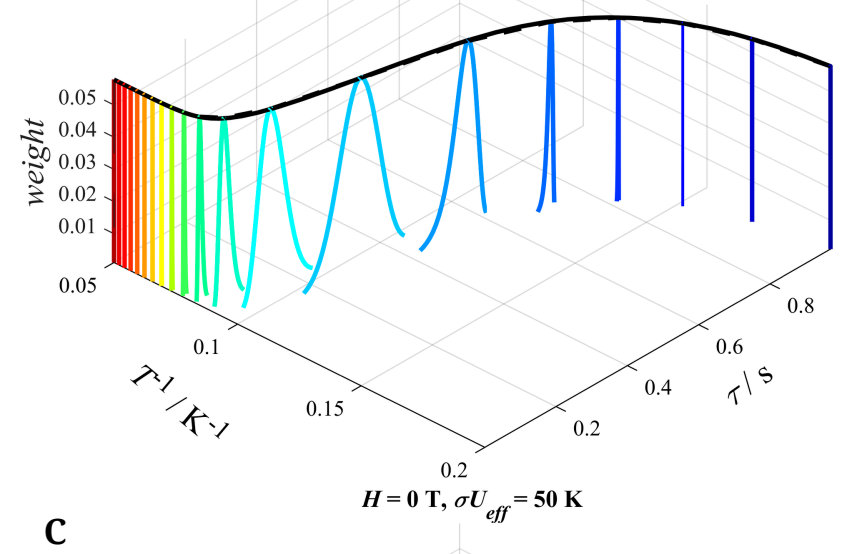

C

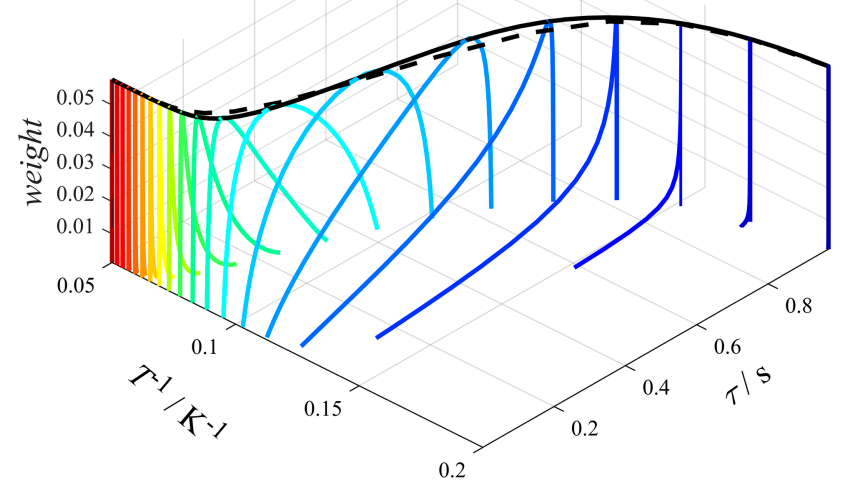

B

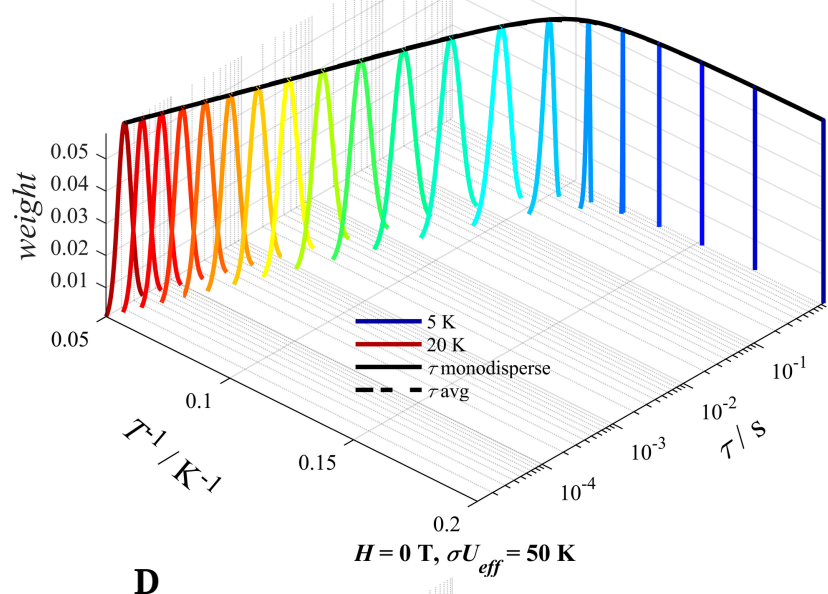

D

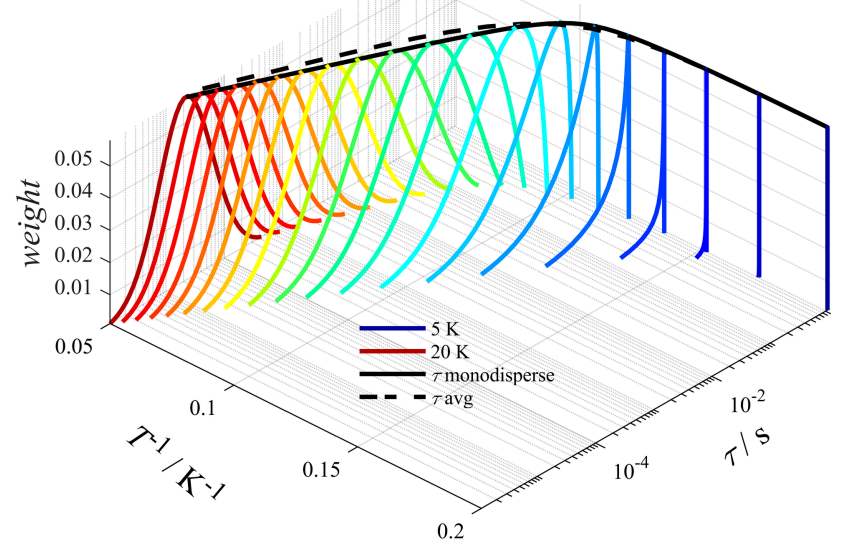

Figure 2. Distribution of relaxation times of the hypothetical SMM at different temperatures 4-12 K, for a Gaussian distribution of $U_{\text {eff }}$ (central value: $200 \mathrm{~K}$ ). (A) For $\sigma U_{\text {eff }}=10 \mathrm{~K}$ (5\% of $U_{\text {eff }}$ ) with a linear $\tau$-axis. (B) For $\sigma U_{\text {eff }}=10 \mathrm{~K}$ ( $5 \%$ of $U_{\text {eff }}$ ) with a logarithmic $\tau$-axis. (C) For $\sigma U_{\text {eff }}=50 \mathrm{~K}$ ( $25 \%$ of $\left.U_{\text {eff }}\right)$ with a linear $\tau$-axis. (D) For $\sigma U_{\text {eff }}=50 \mathrm{~K}$ ( $25 \%$ of $\left.U_{\text {eff }}\right)$ with a logarithmic $\tau$-axis. The black continuous line is the $\tau$ vs $T$ curve of the monodisperse system. The black dashed line is the weighted average $\tau$ curve for the distributed system.

It may be seen that for roughly comparable distributions, the Debye model yields far broader, but also more symmetrical, lognormal $\chi^{\prime \prime}(f)$ peaks. The Gaussian $U_{\text {eff }}$ distribution yields narrower peaks, but whose maximum is more shifted with respect to that of the monodisperse system. Similarly, the Argand plots are more compressed and symmetrical for the former model and more skewed for the latter, reminiscent of the respective plots according to the Cole-Davidson model [11]. 
A

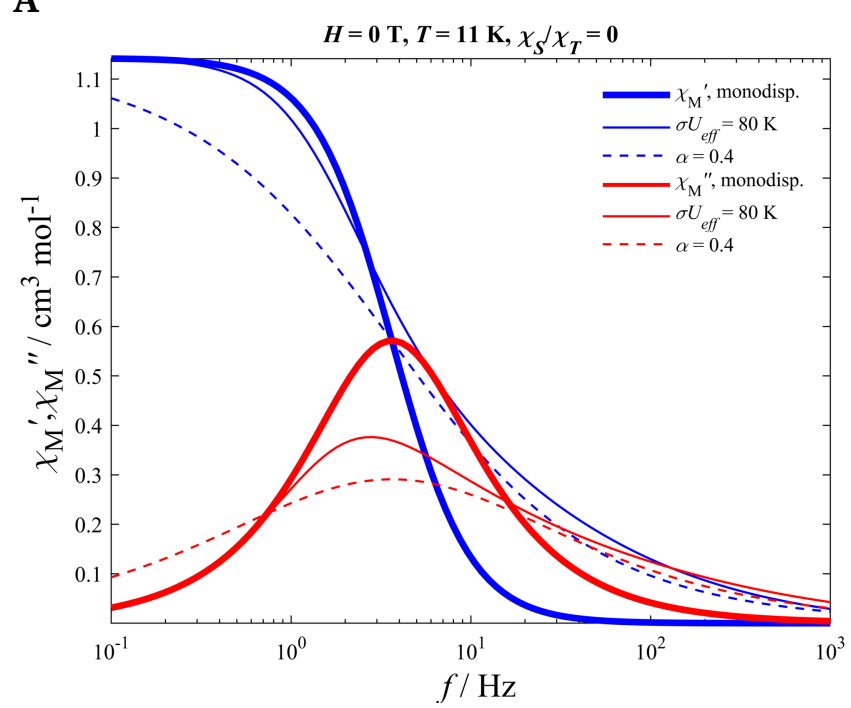

C

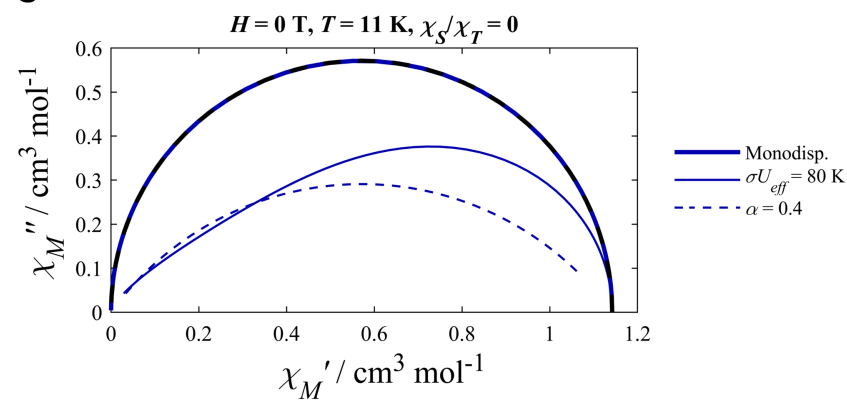

E

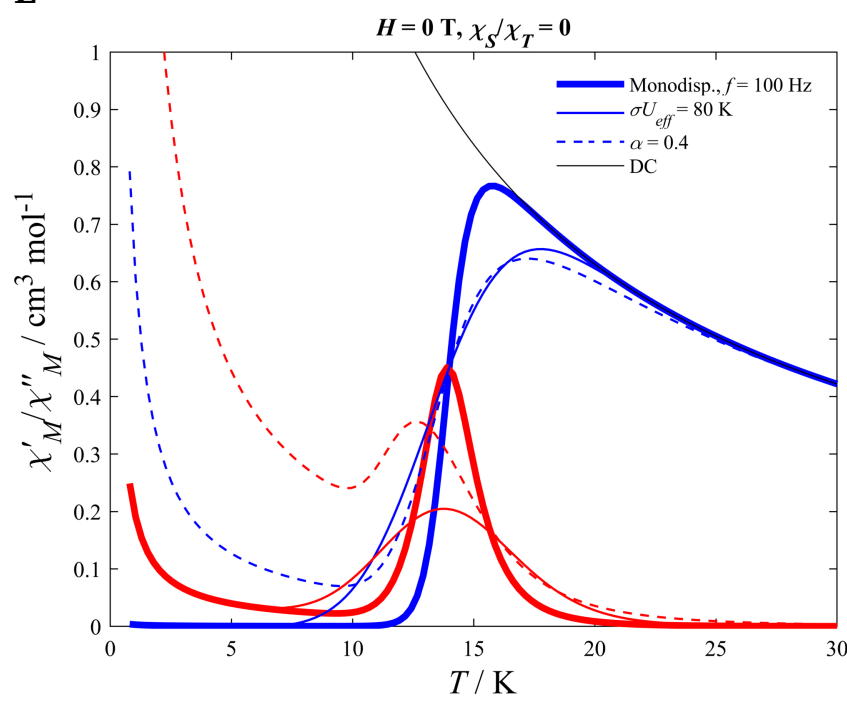

B

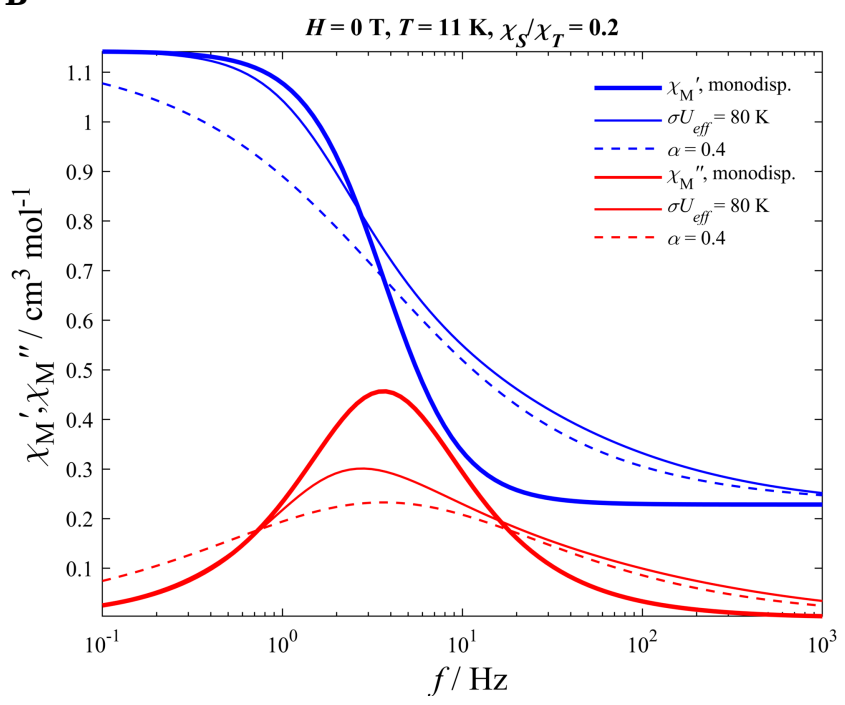

D

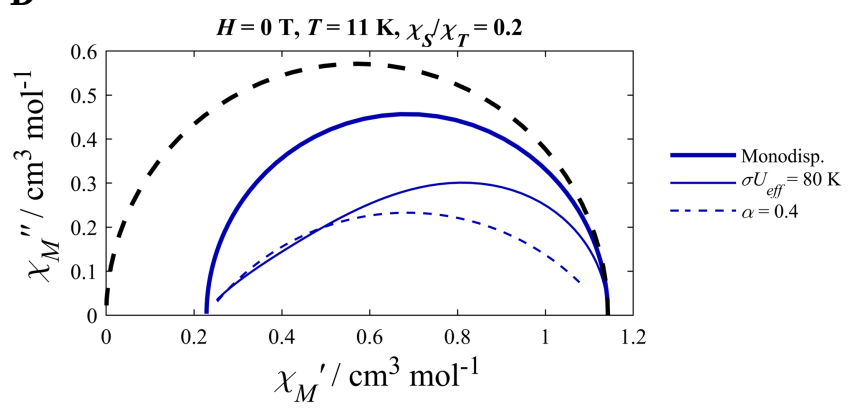

$\mathbf{F}$

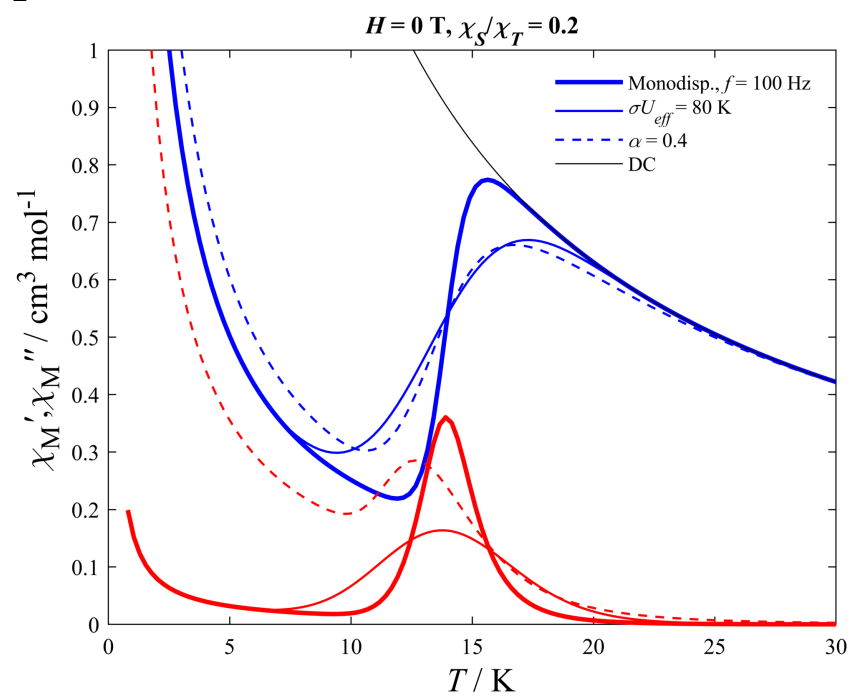

Figure 3. Different representations of the effect of different distribution models on the hypothetical SMM for a fully and partially relaxing fraction (80\%). (A, B) $\chi_{M}{ }^{\prime}$ (blue) and $\chi_{M}{ }^{\prime \prime}$ (red) vs $f$ plots for $\chi_{S} / \chi_{T}=0$ (A) and $\chi_{S} / \chi_{T}=0.2$ (B). (C, D) $\chi_{M}{ }^{\prime \prime}$ vs $\chi_{M}{ }^{\prime}$ plots for $\chi_{S} \chi_{T}=0$ (C) and $\chi_{S} \chi_{\chi_{T}}=0.2$ (D). The black dashed semicircle indicates the fully-relaxing monodispersed system. (E, F) $\chi_{M}{ }^{\prime}$ (blue) and $\chi_{M}{ }^{\prime \prime}$ (red) vs $f$ plots for $\chi_{s} \chi_{T}=0$ (E) and $\chi_{s} / \chi_{T}=$ 0.2 (F). The very thick lines in $\mathbf{A}$ and $\mathbf{E}$ indicate the situation of an ideal, fully relaxing $(\rho=1)$, monodisperse system. Approximately comparable distributions were used, i.e., $\sigma U_{\text {eff }}=0.4 \cdot U_{\text {eff }}$ for the Gaussian distributions and $\alpha=0.4$ for the Debye distribution. The isothermal simulations are at $10 \mathrm{~K}$ and the isofrequency ones are at $100 \mathrm{~Hz}$. The low- $T$ tail in the out-of-phase signal due to the QTM contribution which is quenched in a $0.1 \mathrm{~T}$ field (see Supplementary Materials). 
Similar observations may be made for the $\chi^{\prime}, \chi^{\prime \prime}(T)$ representation, though in this case an additional source of asymmetry is introduced: since $\tau=f(T)$, the temperature scan will modify the relaxation time at each point of the experiment leading to non-ideal out-of-phase peaks as the sample relaxes with the fastest process (smallest $\tau$ ) at each temperature. For the above example, at zero field the Orbach process is dominant down to $17 \mathrm{~K}$, but upon further cooling QTM takes over. Since $\tau_{\text {QTM }}$ is temperature-independent, $\tau$ remains constant below that temperature and relaxation is not blocked any further. However, the magnitudes of $\chi^{\prime}$ and $\chi^{\prime \prime}$ are also a function of $\chi_{T}(T)$, which increases upon cooling. These tails are precisely due to that low- $T$ increase of $\chi_{T}(T)$.

If, however, QTM is suppressed, e.g., by the application of a magnetic field which lifts the degeneracy of the sublevels, $\tau$ may continue to grow as it will be dominated by other processes before QTM becomes dominant. In our example, a $0.1 \mathrm{~T}$ field causes the temperature-dependent Raman and Orbach processes to remain dominant down to $3 \mathrm{~K}$, causing an increase of $\tau$ just as $\chi_{T}(T)$ increases upon cooling. Thus the effect of $\chi_{T}(T)$ is not observed and the low- $T$ tails are suppressed (see Supplementary Materials).

As may be observed, the decrease of the $\chi$ " signal can be modeled with two assumptions: one of a low fraction of slowly relaxing molecules, and another which assumes a distribution of relaxation times. Indeed, both mechanisms predict a decrease of the maximum $\chi^{\prime \prime}$ value, which raises the question as to which is the most relevant in a given case. As may be seen from the above simulations, any of the considered distribution schemes is associated with broadenings of the $\chi^{\prime \prime}(f)$ and $\chi^{\prime \prime}(T)$ peaks. On the contrary, a slowly relaxing fraction $\left(\chi_{S} / \chi_{T}>0\right)$ causes no such broadening and the line width in the $\chi^{\prime \prime}(f)$ representation remains $\Delta f_{\text {FWHM }}$ $=\sqrt{3} / \pi \tau$ (see above), e.g., a $\chi$ " signal of half the ideal intensity could be explained either by assuming a $\rho=0.5$ slowly relaxing fraction, or by a distribution parameter $\alpha \sim 0.41$. In the former case the FWHM would be exactly that of a fully relaxing sample, whereas in the latter it would be almost 4 times larger.

This observation provides a useful heuristic in selecting a model that reproduces weak $\chi "$ signals: if such signals are broader than in the monodisperse system, a generalized distribution may be considered, whereas if they are only weaker in magnitude, only a low relaxing fraction $\left(\chi_{S} / \chi_{T}>0\right)$ is necessary.

\section{Modeling of $M(H(t))$}

As mentioned above, Equation (15) needs to be solved at each point of the field-sweep experiment using the relevant $M_{e q}(T, H)$ and $\tau(T, H)$ parameters for that specific magnetic field. For any magnetic field increment $\Delta H=H(t+\Delta t)-H(t)$, the final magnetization $M(T, H(t+\Delta t))$ can be calculated by using the magnetization $M(T, H(t))$ as the initial condition of the differential equation. It is also considered that at time $t=$ 
0 , at the initial magnetic field $H(t=0)=-H_{\max }$, it holds that $M(T, H(t=0))=$ $-M_{e q}\left(T, H_{\max }\right)$, i.e., at the beginning of the experiment the sample is at its equilibrium magnetization for the corresponding field and temperature.

A numerical solution based on Euler's method was tested by considering that the change in magnetization over the magnetic field increment $\Delta H$ will be $\Delta M=-(1 / \tau(T, H)) \cdot\left[M(T, H(t))-M_{e q}(T, H)\right] \cdot \Delta t$, where $\Delta t$ $=\Delta H / \kappa$, and that the magnetization after time $\Delta t$ would be $M(t+\Delta t)=M(t)$ $+\Delta M$. While this method yielded overall correct results, it was prone to produce discontinuities which, as previously noted [4], require additional treatment, such as smoothing.

On the contrary, numerical solution using the Runger-Kutta method implemented by Matlab's embedded solvers (ode45 in particular), consistently produced realistic curves without additional treatment. Although this adds a slightly higher computational overhead with respect to the previous method, the higher stability and accuracy it affords make it an uncontested choice for this application.

Up till now, it is assumed that each interval $\Delta H$ of the ascending magnetic field is swept a rate $\kappa=\mathrm{d} H / \mathrm{d} t$ common for all intervals, which is equivalent with assuming a constant field-sweep rate. It has been commented [50] that this numerical solution is applicable to methods that measure the magnetization continuously as the field is swept, such as with micro-SQUID or VSM magnetometers, in which case the experimental sweep rate coincides with the numerical one. The situation is distinctly different from conventional SQUID magnetometers, which require a field stabilization before a measurement is carried out.

To simulate conventional SQUID experiments, still available in many labs, an experimental delay time $\delta$ is also considered, which corresponds to the duration of the process entailing magnetic field stabilization at its final value and the actual measurement. Thus, the initial condition to calculate the magnetization at $M(T, H+\Delta H)$ becomes $M_{e q}(T, H)+[M(T$, $\left.H(t))-M_{e q}(T, H)\right] \mathrm{e}^{-\delta / \tau(T, H)}$, which accounts for an additional relaxation of the magnetization with a relaxation time $\tau(T, H)$ over time $\delta$. Moreover, since such experiments are often carried out over inhomogeneous domains (more concentrated data appoints at low fields), to better account for real experimental protocols, SMM-ET can also consider field sweeps with non-equidistant data points.

Simulations of the hypothetical SMM presented above reveal, as expected, steps at the level-crossing fields and a coalescence of the hysteresis loops to the equilibrium curve as $\rho \rightarrow 0$ (Figure 4). Also, the increase of the delay time $\delta$ is clearly manifested as a narrowing of the hysteresis and a smoothening of the QTM-induced steps.

The usefulness of this functionality extends to revealing relaxation characteristics that are not usually obvious from isothermal/isofrequency ac susceptibility experiments carried out at zero magnetic field, or at only a few magnetic fields (see below). 
A

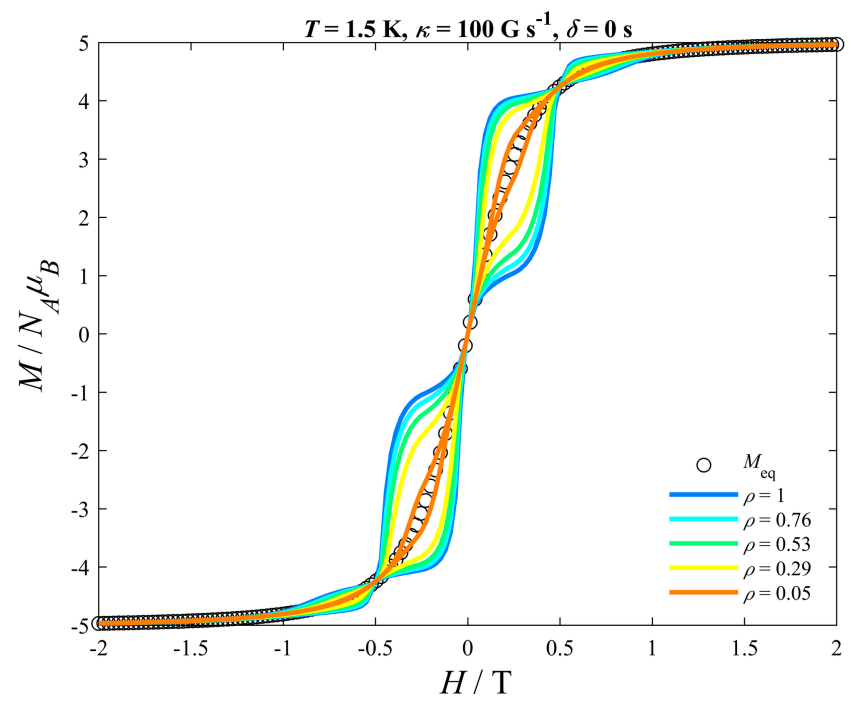

B

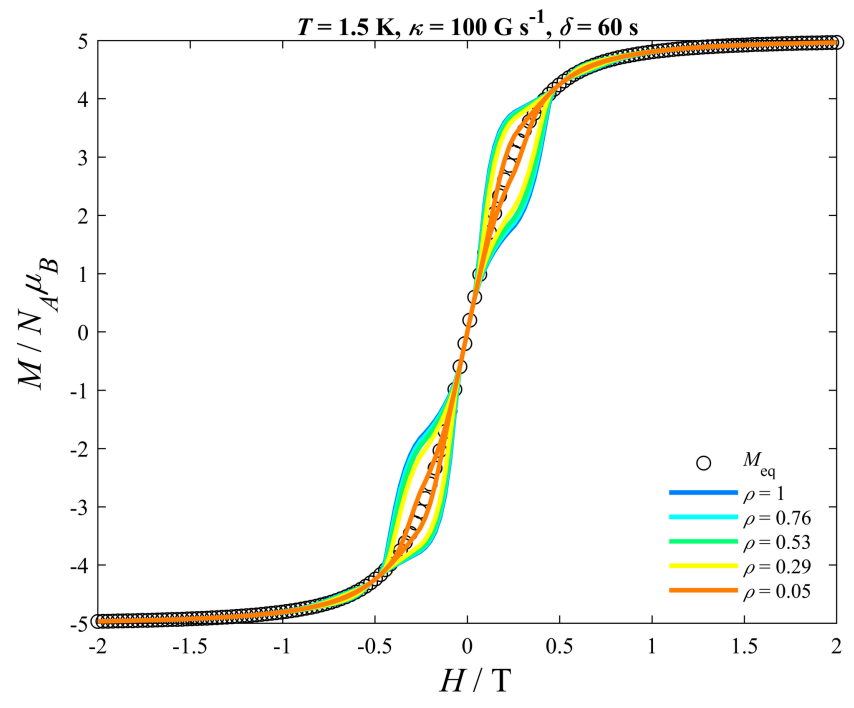

Figure 4. Magnetic hysteresis loops of the hypothetical Dy ${ }^{\mathrm{III}} \mathrm{SMM}$ at $1.5 \mathrm{~K}$, with the magnetic relaxation parameters of Figure 1 and an additional level crossing at $0.5 \mathrm{~T}$. The loops are calculated for 150 points per branch at a scan rate of $0.1 \mathrm{~T} \mathrm{~s}^{-1}$. (A) Assuming an "on the fly" experiment typical of VSM or micro-SQUID devices. (B) Assuming a "stop and measure" experiment typical of conventional SQUID devices, with a measuring delay of $60 \mathrm{~s}$ per point. The various curves correspond to different slowly relaxing fractions.

\section{Examples of Real Systems}

In this section we will consider three SMM examples, characterized as "strong", "medium" and "weak", depending on the intensity of their out-of-phase signals. For the two former a parametric reproduction of the $\chi_{T}(T, H)$ curves was possible based on bibliographic crystal-field parameters (CFPs), whereas for the latter, the size of the system precluded any such calculation, allowing the demonstration of the interpolation method.

Example 1: a "strong" Dy"II SMM

For this example we consider complex $\left[\left(\eta^{5}-\mathrm{Cp} *\right) \operatorname{Dy}\left(\eta^{5}-\mathrm{Cp} \mathrm{p}^{\mathrm{iPr} 5}\right)\right]\left[\mathrm{B}\left(\mathrm{C}_{6} \mathrm{~F}_{5}\right)_{4}\right]$ (1) [48], an organometallic double-decker SMM, characterized by a record magnetization reversal barrier of $2217 \mathrm{~K}$. This complex exhibits discernible magnetic hysteresis up to $\sim 80 \mathrm{~K}$ and strong out-of-phase signals. Fits to dc magnetization relaxation (2-82 K) and ac-susceptibility $(83-138 \mathrm{~K})$ experiments yielded magnetic relaxation parameters: $U_{\text {eff }}=$ $1541 \mathrm{~cm}^{-1}(2217 \mathrm{~K}), b_{\text {Orbach }}=1 / 4.2 \times 10^{-12} \mathrm{~s}^{-1}=2.4 \times 10^{11} \mathrm{~s}^{-1}, n=3, b_{\text {Raman }}=$ $3.1 \times 10^{-8} \mathrm{~s}^{-1} \mathrm{~K}^{-3}, b_{\text {Direct }}=0, b_{\mathrm{QTM} 1}=1 / 2.5 \times 10^{4} \mathrm{~s}^{-1}=4.0 \times 10^{-5} \mathrm{~s}^{-1}, b_{\mathrm{QTM} 2}=0$. These reproduce very well the ac susceptibility experimental data in simulations carried out with the SMM evaluation tool, and are presented without further processing or refitting, unless otherwise stated.

In Figure 5 are shown indicative simulations of the isofrequency data at $31.50 \mathrm{~Hz}$, assuming fully $(\rho=1)$ and partially relaxing samples. These simulations confirm that this is a "strong" but not perfect SMM, with 
$\sim 96.5 \%$ of the sample undergoing slow magnetic relaxation. As such they serve as control simulations of the SMM evaluation tool.

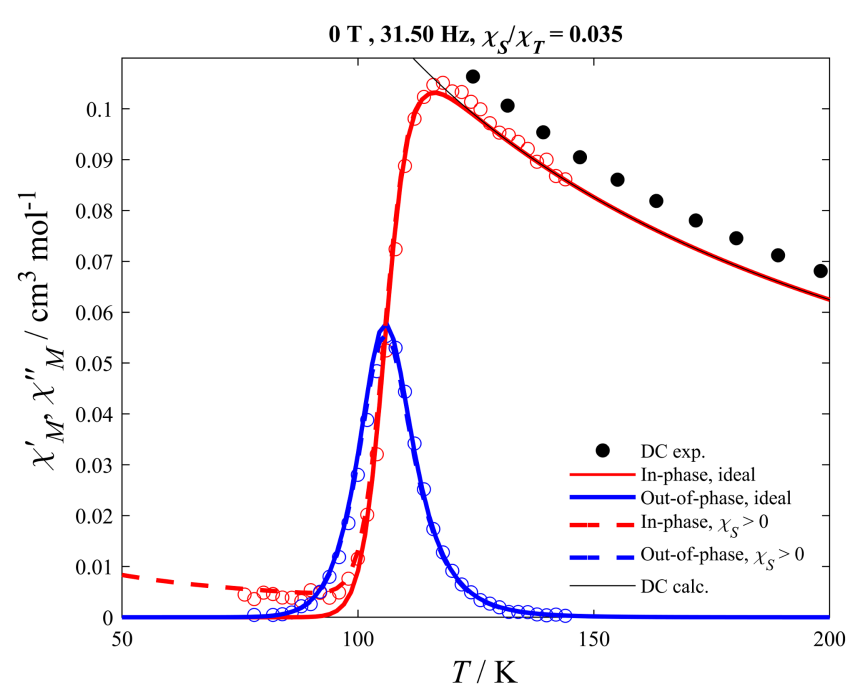

Figure 5. $\chi_{M}^{\prime}, \chi_{M}{ }^{\prime \prime}$ vs $T$ experimental data for 1 at zero magnetic field and at a frequency of $31.50 \mathrm{~Hz}$ (red, blue open circles) and simulations (red, blue lines) based on the literature relaxation parameters. The black line is a parametrically calculated curve based on the CFPs, properly scaled to agree with the ac data. Black circles represent DC experimental data. The consideration of a $96.5 \%$ slowly relaxing fraction $\left(\chi_{S} / \chi_{T}=\right.$ 0.035) accounts for the small tail of the in-phase signal below $100 \mathrm{~K}$ and is consistent with the values derived from fits to Argand plots.

We also use this SMM to test the possibility to refine the magnetic relaxation parameters from field-sweep experiments. Field sweep "on the fly" experiments have been reported for this complex at $2 \mathrm{~K}$ and at various scan rates using a VSM magnetometer. Due to the large number of data points of that data set which preclude a full-matrix calculation of the equilibrium magnetization at each point, this study is ideal to present the use of interpolated such curves. Since $M$ vs $H$ dc studies were not reported for this complex [48], to illustrate this point, the respective data of a similar complex (complex 2 in that work) were used to create the interpolated curve.

Using the above mentioned parameters in the simulation of the hysteresis curves, it is observed that the predicted loops are narrower that the experimental ones, whereas the step near zero field is not reproduced. Adding terms that are associated with field-dependent processes, it is possible to better approach the form of the hysteresis loops, in particular using $b_{\text {Direct }} 3.6 \times 10^{-9} \mathrm{~s}^{-1} \mathrm{~K}^{-1} \mathrm{~T}^{-4}$ and $b_{\text {QTM2 }}=10^{6} \mathrm{~T}^{-2}$ (Figure 6, see Equation (16) for the meaning of the terms). This improvement in the simulation of variable-field experiments is to be expected, since the originally reported kinetic parameters are extracted from static-field experiments (zero-field ac susceptibility), which contain less information regarding field-dependent processes. 


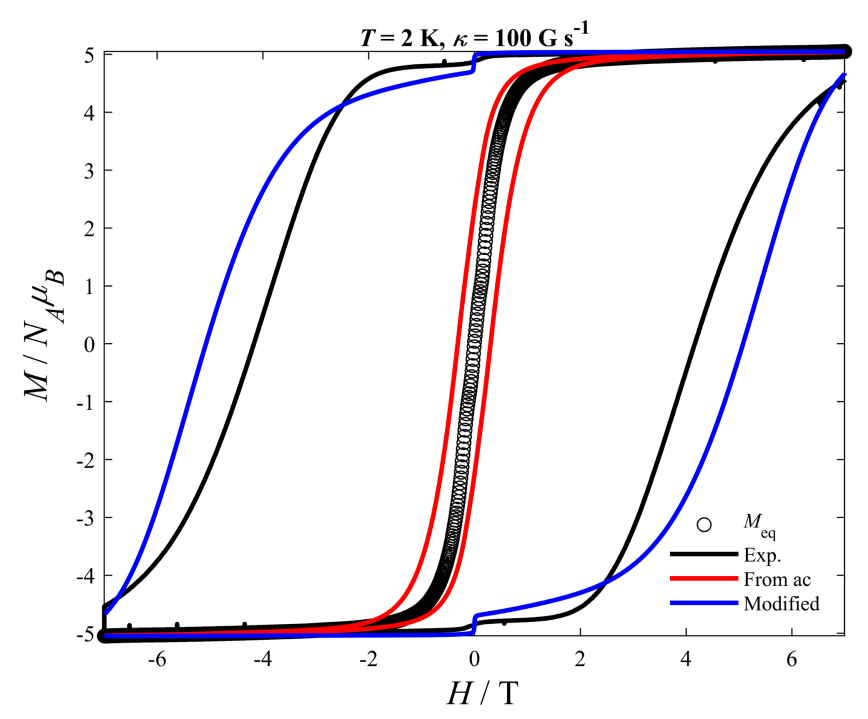

Figure 6. Hysteresis loop of 1 at a scan rate of $100 \mathrm{G} \mathrm{s}^{-1}$ and simulations based on the literature relaxation parameters derived from ac susceptibility and magnetization relaxation data (red line) and on the additional consideration of $b_{\text {Direct }}=3.5 \times 10^{-9} \mathrm{~s}^{-1} \mathrm{~K}^{-1} \mathrm{~T}^{-4}, b_{Q T M 2}=10^{6} \mathrm{~T}^{-2}, H_{Q T M}=0$ (blue line). In the absence of $M$ vs $H$ dc data for this complex, the equilibrium magnetization curve $M_{e q}(\circ)$ was calculated from interpolation of a similar complex from the same work (complex 2 of reference [48]) to the domain of the field-sweep data (black line).

\section{Example 2: a "medium" Dy III SMM}

Complex $\left[\mathrm{Fe}_{2}{ }_{2} \mathrm{Dy}^{\mathrm{III}}{ }_{2}(\mathrm{mepao})_{6}(\mathrm{mepaoH})_{2}(\mathrm{NCS})_{4}\right](2)[51]$ is a tetranuclear 3d-4f SMM, containing two low-spin $(S=0) \mathrm{Fe}^{\mathrm{III}}$ ions, leaving the Dy ${ }^{\mathrm{III}}$ ions as the only magnetic moment carriers of the complex. Analysis of its ac data has yielded kinetic parameters $U_{\text {eff }}=40 \mathrm{~K}, b_{\text {Orbach }}=1 / 2.6 \times 10^{-8} \mathrm{~s}^{-1}=$ $3.8 \times 10^{7} \mathrm{~s}^{-1}, n=3.03, b_{\text {Raman }}=0.05748 \mathrm{~s}^{-1} \mathrm{~K}^{-3.03}, b_{\text {Direct }}=0, b_{\mathrm{QTM} 1}=1 / 5.6 \times 10^{-4}$ $\mathrm{s}^{-1}=1.8 \times 10^{3} \mathrm{~s}^{-1}, b_{\mathrm{QTM} 2}=0$.

This SMM is characterized by weak out-of-phase signals, which do not cross with the in-phase ones (Figure 7), an attribute characteristic of $\chi_{s}>$ $0(\rho<1)$. Moreover, they exhibit a characteristic low-temperature tail, indicative of QTM becoming dominant at low temperatures (see above). An initial attempt to reproduce these data gave improved agreement when considering a value of $\chi_{S} / \chi_{T}=0.4(\rho=0.6)$, indicating a $60 \%$ of slowly relaxing fraction. Additional improvement was achieved by slightly modifying the value of the $b_{Q T M 1}$ parameter from $5.6 \times 10^{-4}$ to $4.0 \times$ $10^{-4} \mathrm{~s}^{-1}$. Of course, this may not be the only combination of kinetic parameters that similarly improves the agreement, but it was deemed preferable not to introduce additional relaxation mechanisms to the model. 


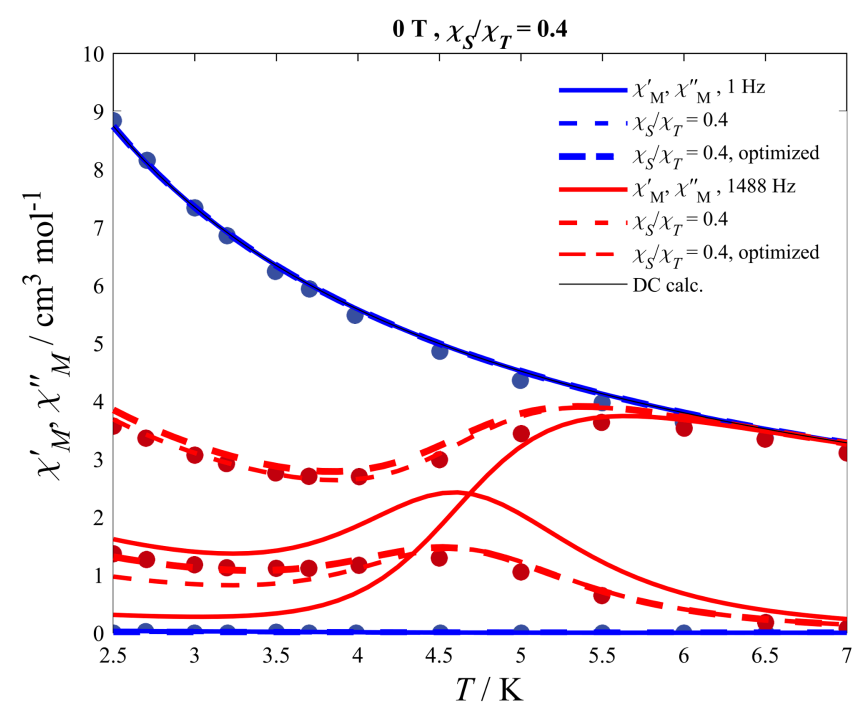

Figure 7. Experimental ac data of 2 at 1 and $1488 \mathrm{~Hz}$ and simulations: $1 \mathrm{~Hz}$, blue points/lines; $1488 \mathrm{~Hz}$, red points/lines. The continuous lines correspond to a fully relaxing sample and the thin dashed lines to a $60 \%$ relaxing sample $\left(\chi_{S} / \chi_{T}=0.4\right)$. The thick dashed lines correspond to an optimized relaxation time assuming $b_{Q T M 1}=4.0 \times 10^{-4}$ instead of $5.6 \times 10^{-4} \mathrm{~s}^{-1}$.

Example 3: a "weak" $\mathrm{Fe}^{I I}{ }_{9} S M M$

Complex $\left[\mathrm{Fe}_{9}(\mathrm{NCO})_{2}\left(\mathrm{O}_{2} \mathrm{CMe}\right)_{8}\left\{(2-\mathrm{py})_{2} \mathrm{CO}_{2}\right\}_{4}\right]$ (3) is a ferromagnetic ferrous cluster previously reported by this author as one of the first ferrous SMMs [52]. It may be noted that the out-of-phase signals are weak, although they are quite sharp and distinctive. This strongly points toward a low fraction of slowly relaxing molecules rather than a distribution of relaxation times. Thus, this molecule is an excellent candidate for a quantitative treatment of its ac susceptibility data.

In implementing the previously outlined methodology, it quickly becomes clear that the mere size of the full spin Hamiltonian matrix of 3 $\left[(2 \times 2+1)^{9}=1,953,125\right]$ precludes any possibility for a parametric calculation of its dc magnetic susceptibility curve. For the high- $T$ susceptibility of the azido analogue of $\mathbf{3}$ the Kambe vector coupling scheme has been used, facilitated by the fortuitous symmetry of the molecule [53]. However, this calculation fails to account for the low- $T$ region, governed by single-ion zfs, and at which slow magnetic relaxation occurs. Thus, this system is doubly instructive, as its treatment illustrates the interpolative reproduction of the dc susceptibility curve for the quantitative calculation of ac susceptibilities.

For this calculation, the $1 \mathrm{kG}$ dc data were used to construct an approximation of the low- $T \chi_{T}(T)$ function. Actually, this curve agrees quite well with the high- $T$ part of the zero-field $\chi^{\prime}{ }_{M}$ signal (Figure 8) lending credence to the subsequent treatment. Using the parameters from the Orbach relaxation mechanism, the position of the $\chi^{\prime \prime}{ }_{M}$ peak is nicely reproduced, whereas its magnitude is seriously overestimated. At the same time, the calculated magnitude of $\chi_{M}^{\prime}$ (shown in the figure as $\chi_{M}^{\prime} T$ ) predicts a sharp drop not observed in the experimental data. By introducing an adiabatic susceptibility such that $\chi_{S} / \chi_{T}=0.8(20 \%$ slowly 
relaxing fraction) the magnitudes of the calculated in- and out-of-phase components nicely reproduce those of the experimental ones. Some additional improvement is also possible by assuming a weak distribution of the relaxation times, either as an empirical Debye parameter $(\alpha=0.1)$ or as a Gaussian distribution of the magnetic reversal barried $\left(\sigma U_{\text {eff }}=\right.$ $0.1 U_{\text {eff }}$ ). In either case, attempts to entirely account for the weak out-of-phase signal using a distribution model produce unrealistically broad curves. As the signal is weak but also quite narrow, a low slowly relaxing fraction is suited to account for its small magnitude, without introducing extreme broadenings.

A

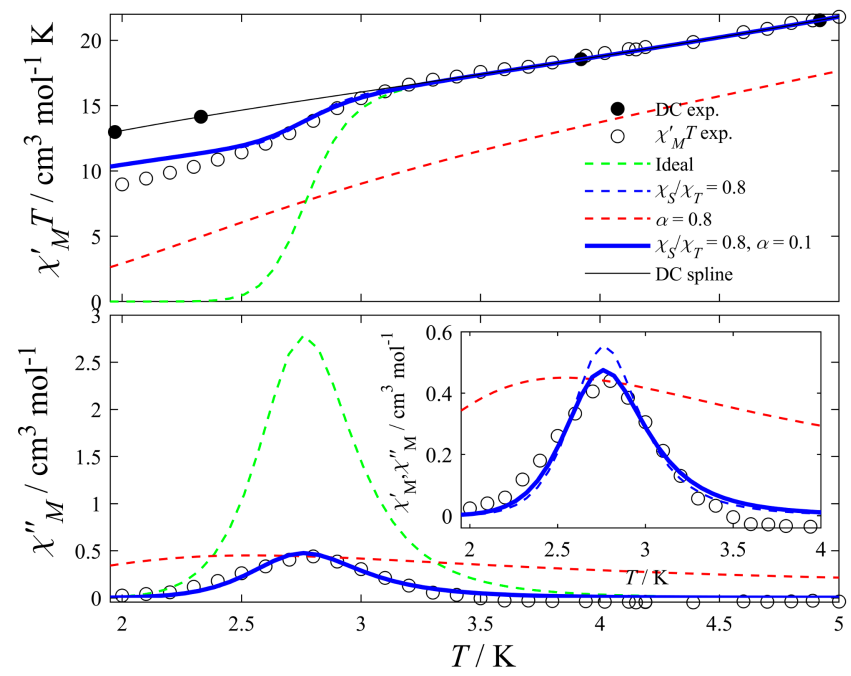

B

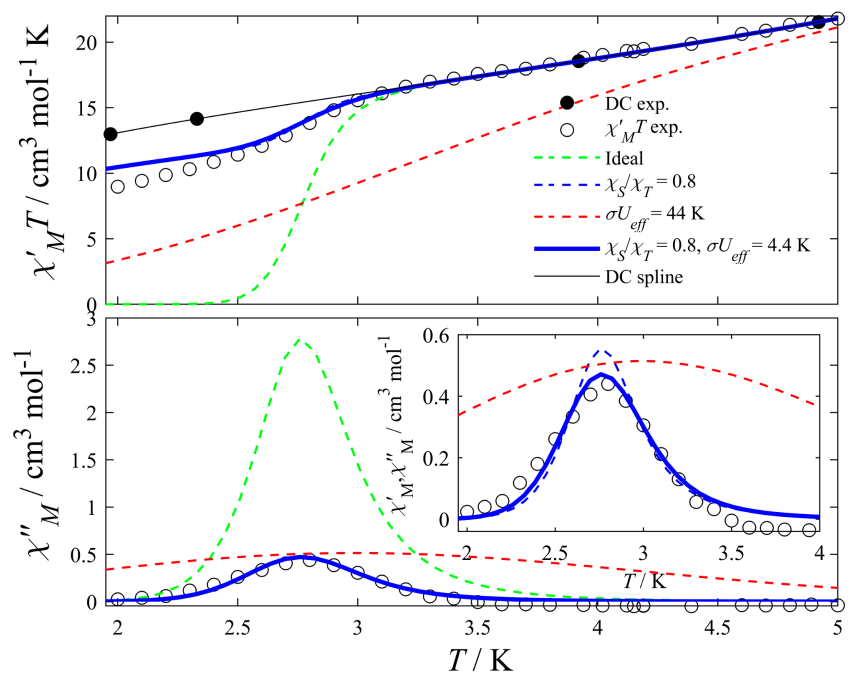

Figure 8. Experimental $\chi_{M}{ }^{\prime} T$ vs $T$ (top) and $\chi_{M}{ }^{\prime \prime}$ vs $T$ (bottom) ac data of 3 under a $1000 \mathrm{~Hz}$ oscillating field and their simulations according to different models. (A,B) Dashed green line $(--)$ : fully relaxing sample with a unique relaxation time. Dashed blue line (--): low relaxing fraction (20\%) with a unique relaxation time. (A) Dashed red line (--): fully relaxing sample with a large Debye distribution $(\alpha=0.8)$ to account for the low $\chi_{M}$ " signal. Thick blue line (-): low relaxing fraction (20\%) with is a small Debye distribution $(\alpha=$ 0.1). (B) Dashed red line (- $)$ : fully relaxing sample with a large $U_{\text {eff }}$ distribution $\left(\sigma U_{\text {eff }}=U_{\text {eff }}=44 \mathrm{~K}\right)$ to account for the low $\chi_{M}$ " signal. Thick blue line (-): low relaxing fraction (20\%) with is a small $U_{\text {eff }}$ distribution $\left(\sigma U_{\text {eff }}=U_{\text {eff }} / 10=4.4 \mathrm{~K}\right)$. The insets are enlargements of the out-of-phase signals.

\section{IMPLEMENTATION}

In order to benefit from additional functionality, the tool has been written in Matlab and can make use of Easyspin's curry function to parametrically calculate dc susceptibilities and magnetizations. However, any experimental dataset or any calculated curve generated by Phi or any other program can also be used instead. In that case though, care must be taken in defining the $T$ and $H$ domains so that they overlap with those of the ac/hysteresis data to be simulated by the tool. SMM-ET will then create new dc datasets through interpolation with Matlab's cubic spline function. This capability may also be useful when a parametric calculation is possible but computationally expensive. 
In its current version, SMM-ET is available as a Matlab executable (SMM_ET.p) which requires a Matlab working environment to run, as well as Easyspin if curry is to be invoked. The parameters of the SMM and of the experiments to be simulated are given in a required text file (SMM_params.dat) that must be present in the executable's path. If the dc properties of the SMM are to be parametrically simulated, the spin system has to be defined in Easyspin format using "Sys" as structure name (Sys.g, Sys.D, etc.), with the structure saved as a Matlab file (SMM_Sys.mat).

Experimental dc data (or curves calculated with other software, e.g., Phi) need to be provided as ascii files (chi_Vs_T_dc.dat and M_vs_H_dc.dat). These are optional, but if they are present in the executable path they will override the Easyspin calculation. It should be noted that just one of those may be provided and only the respective calculation ( $\chi_{\mathrm{M}}$ vs $T$ or $M$ vs $H$ ) will be overridden. In addition, magnetic hysteresis simulations can be carried out for experiments with non-equidistant data points. In that case, and assuming that the ascending and descending branches are symmetrical, an ascii file with the experimental field positions of only the ascending branch, i.e., from $-H_{\max }$ to $+H_{\max }$ can be supplied (M_vs_H_sweep.dat). This file only needs to contain the magnetic field positions (not the magnetizations). If present, it will override the magnetic field domain given in SMM_params.dat.

In its current version, SMM-ET outputs the calculated curves in a series of self-explanatory figures. The data of each curve will appear on the workspace under self-explanatory names. However, users can also extract the data from the figures using the Matlab command line.

More detailed instructions and the tool executable (along with a sample parameter file) are provided at http://chiralqubit.eu/

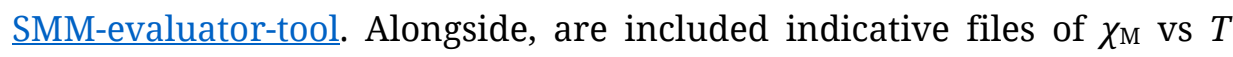
and $M$ vs $H$ experimental data [48] of Dy ${ }^{\text {III SMMs. }}$

\section{DISCUSSION}

The quantitative analysis of the ac susceptibilities presented above illustrates the additional amount of information available from isothermal and isofrequency ac experiments. In particular, an important parameter that is rarely addressed is the absolute intensity of the out-of-phase signals and its relation to the slowly relaxing fraction of the sample. Even if distributions of the relaxation times have been properly considered for the slowly relaxing fraction, they cannot by themselves account for very weak out-of-phase signals. Indeed, the characteristic examples of a "medium" SMM (complex 2, 60\% slowly relaxing fraction) and a "weak" SMM (complex 3, 20\% slowly relaxing fraction) oblige us to address the fundamental reasons behind such low fractions of slowly relaxing samples. 
Looking toward an intramolecular mechanism to account for this, in particular Orbach relaxation over the spin reversal barrier $U_{\text {eff, models }}$ assuming even unrealistically broad distributions were clearly shown to be inadequate in explaining such experimental signatures. Also staying within the intramolecular context, QTM cannot fully account for such a situation either. Indeed, low out-of-phase signals can still be the case even under dc magnetic fields aimed at suppressing QTM [54-58].

It is therefore clear that we need to look beyond the Orbach and QTM processes. Indeed, it has been pointed out that when any process becomes too slow at a particular temperature and magnetic field, relaxation will simply occur through another mechanism which is faster at these conditions. Intermolecular pathways involving vibronic degrees of freedom and coupling to the environment through the phonon bath (and eventually the conducting electron bath of metallic substrates) need to be accounted for to reproduce the full relaxation profiles of SMMs [59].

To obtain information on such processes based on bulk ac susceptometry data of pure samples, it is noted here that $\chi_{s}$ should be viewed as a statistical parameter which characterizes the sample as a whole. In the context of pure bulk samples, it so happens that the molecule is also its own environment, in the sense that each molecule neighbors identical, or almost identical molecules; so $\chi_{s}$ could be loosely considered to characterize the molecule, but only for a particular crystal form. However, when we consider SMM samples in drastically different environments, e.g., surface-deposited molecules, this analogy would be more tenuous. In such a case, $\chi_{s}$ might eventually be used to describe the system of a single molecule and its environment, although it is more probable that the development of another theoretical framework would be required.

Recently, it was discovered that the magnetization of SMMs is better stabilized when they are deposited on insulating substrates with low phonon density of states (e.g., MgO) than when deposited on metallic surfaces; actually, such samples are better stabilized even with respect to the pure bulk samples. It was found that this stabilization is related to the more efficient blocking of phonon-induced relaxation pathways $[4,60]$. Such realizations reinforce the understanding that the environment of SMMs is not innocent in determining their magnetic relaxation, which means that molecular engineering needs to address this reality, in addition to targeting the maximization of the magnetization reversal barrier.

The quantitative study of ac magnetic data such as described here could provide us with additional information on such processes, in a way which is simple, cost effective, and easy to apply routinely on a large number of samples. Of course, this information would need to be complemented by theoretical calculations and dedicated studies for a better understanding of the behavior of specific molecules. However, taken over large sample numbers it could provide just as valuable 
conclusions as these more laborious and costly studies, in the way that large datasets of magnetic exchange couplings helped magnetochemists set the basis for an empirical understanding of magnetostructural correlations.

\section{SUPPLEMENTARY MATERIALS}

The following supplementary materials are available online at https://doi.org/10.20900/qmr20200004, Figure S1: Distributions of relaxation times under a $0.1 \mathrm{~T}$ magnetic field which suppresses QTM, Figure S2: Effect of different distribution models on the hypothetical SMM for a fully and partially relaxing fraction (80\%) under a $0.1 \mathrm{~T}$ field which suppresses QTM; and a Zip file with program executable and sample parameter files.

\section{CONFLICTS OF INTEREST}

The author declares that there is no conflict of interest.

\section{FUNDING}

This project has received funding from the Fondation pour la Recherche en Chimie (Strasbourg) under the Innovation grant agreement PTu-FRC-0003 (project "MiSSTri”).

\section{ACKNOWLEDGMENTS}

The author thanks Professor Philippe Turek for a critical reading of this manuscript.

\section{REFERENCES}

1. Caneschi A, Gatteschi D, Sessoli R, Barra AL, Brunel LC, Guillot M. Alternating current susceptibility, high field magnetization, and millimeter band EPR evidence for a ground $S=10$ state in $\left[\mathrm{Mn}_{12} \mathrm{O}_{12}\left(\mathrm{CH}_{3} \mathrm{COO}\right)_{16}\left(\mathrm{H}_{2} \mathrm{O}\right)_{4}\right] \cdot 2 \mathrm{CH}_{3} \mathrm{COOH} \cdot 4 \mathrm{H}_{2} \mathrm{O}$. J Am Chem Soc. 1991 Jul;113(15):5873-4.

2. Sessoli R, Gatteschi D, Caneschi A, Novak MA. Magnetic bistability in a metal-ion cluster. Nature. 1993 Sep;365(6442):141-3.

3. Atkinson JH, Park K, Beedle CC, Hendrickson DN, Myasoedov Y, Zeldov E, et al. The effect of uniaxial pressure on the magnetic anisotropy of the $\mathrm{Mn}_{12}-\mathrm{Ac}$ single-molecule magnet. EPL Europhys Lett. 2013 May 1;102(4):47008.

4. Studniarek M, Wäckerlin C, Singha A, Baltic R, Diller K, Donati F, et al. Understanding the Superior Stability of Single-Molecule Magnets on an Oxide Film. Adv Sci. 2019 Sep 30;6(22):1901736.

5. Chiorescu I, Wernsdorfer W, Müller A, Bögge H, Barbara B. Butterfly Hysteresis Loop and Dissipative Spin Reversal in the $S=1 / 2$, V 15 Molecular Complex. Phys Rev Lett. 2000 Apr 10;84(15):3454-7. 
6. Chen L, Ramsey CM, Dalal NS, Ren T, Cotton FA, Wernsdorfer W, et al. Phonon-bottleneck enhanced magnetic hysteresis in a molecular paddle wheel complex of $\mathrm{Ru}_{2}{ }^{5+}$. Appl Phys Lett. 2006 Dec 18;89(25):252502.

7. Ortu F, Reta D, Ding Y-S, Goodwin CAP, Gregson MP, McInnes EJL, et al. Studies of hysteresis and quantum tunnelling of the magnetisation in dysprosium(III) single molecule magnets. Dalton Trans. 2019;48(24):8541-5.

8. $\quad$ Ding Y-S, Yu K-X, Reta D, Ortu F, Winpenny REP, Zheng Y-Z, et al. Field- and temperature-dependent quantum tunnelling of the magnetisation in a large barrier single-molecule magnet. Nat Commun. 2018 Dec;9(1):3134.

9. Casimir HBG, du Pré FK. Note on the thermodynamic interpretation of paramagnetic relaxation phenomena. Physica. 1938 Jun;5(6):507-11.

10. Gatteschi D, Sessoli R, Villain J. Molecular nanomagnets. Oxford (UK): Oxford University Press; 2006. 395 p. (Mesoscopic physics and nanotechnology series).

11. Topping CV, Blundell SJ. A.C. susceptibility as a probe of low-frequency magnetic dynamics. J Phys Condens Matter. 2019 Jan 9;31(1):013001.

12. Bloch F. Nuclear Induction. Phys Rev. 1946 Oct 1;70(7-8):460-74.

13. Fick E, Sauermann G. The quantum statistics of dynamic processes. Berlin (Germany); New York (US): Springer-Verlag; 1990. 395 p. (Springer series in solid-state sciences).

14. Boudalis AK, Pissas M, Raptopoulou CP, Psycharis V, Abarca B, Ballesteros R. Slow Magnetic Relaxation of a Ferromagnetic $\mathrm{Ni}^{\mathrm{II}_{5}}$ Cluster with an $S=5$ Ground State. Inorg Chem. 2008 Nov 17;47(22):10674-81.

15. Larionova J, Clérac R, Boury B, Le Bideau J, Lecren L, Willemin S. Structural and magnetic studies of the $\left[\mathrm{Mn}_{12} \mathrm{O}_{12}\left(\mathrm{CH}_{3} \mathrm{COO}\right)_{16}\left(\mathrm{H}_{2} \mathrm{O}\right)_{4}\right] \cdot 2 \mathrm{CH}_{3} \mathrm{COOH} \cdot 4 \mathrm{H}_{2} \mathrm{O}$ thermal derivatives. J Mater Chem. 2003 Mar 19;13(4):795-9.

16. Willemin S, Donnadieu B, Lecren L, Henner B, Clérac R, Guérin C, et al. Synthesis and characterization of magnetic organic-inorganic nanocomposites based on the $\left[\mathrm{Mn}_{2} \mathrm{O}_{12}\left\{\mathrm{CH}_{2} \mathrm{C}\left(\mathrm{CH}_{3}\right) \mathrm{COO}_{16}\left(\mathrm{H}_{2} \mathrm{O}_{4}\right]\right.\right.$ building block. New J Chem. 2004;28(8):919-28.

17. Palacio F, Oliete P, Schubert U, Mijatovic I, Hüsing N, Peterlik H. Magnetic behaviour of a hybrid polymer obtained from ethyl acrylate and the magnetic cluster $\mathrm{Mn}_{12} \mathrm{O}_{12}$ (acrylate) 16 . J Mater Chem. 2004;14(12):1873-8.

18. Zhao H, Berlinguette CP, Bacsa J, Prosvirin AV, Bera JK, Tichy SE, et al. Structural Characterization, Magnetic Properties, and Electrospray Mass Spectrometry of Two Jahn-Teller Isomers of the Single-Molecule Magnet $\left[\mathrm{Mn}_{12} \mathrm{O}_{12}\left(\mathrm{CF}_{3} \mathrm{COO}\right)_{16}\left(\mathrm{H}_{2} \mathrm{O}\right)_{4}\right]$. Inorg Chem. 2004 Feb;43(4):1359-69.

19. Gómez-Segura J, Lhotel E, Paulsen C, Luneau D, Wurst K, Veciana J, et al. Trihaloacetic acids: an investigation of steric and inductive ligand effects on the synthesis of $\left[\mathrm{Mn}_{12} \mathrm{O}_{12}\left(\mathrm{O}_{2} \mathrm{CCX}_{3}\right)_{16}\left(\mathrm{H}_{2} \mathrm{O}\right)_{4}\right]$ single-molecule magnets. New J Chem. 2005;29(3):499-503.

20. Gómez-Segura J, Campo J, Imaz I, Wurst K, Veciana J, Gerbier P, et al. New insights into the thermal stability of $\mathrm{Mn}_{12}$ clusters: The case of complex $\left[\mathrm{Mn}_{12} \mathrm{O}_{12}\left(\mathrm{O}_{2} \mathrm{CC} \equiv \mathrm{CH}\right)_{16}\left(\mathrm{H}_{2} \mathrm{O}_{4}\right] \cdot 3 \mathrm{H}_{2} \mathrm{O}\right.$ and its thermolysis derived $\left[\mathrm{Mn}_{3}\left(\mathrm{O}_{2} \mathrm{CC} \equiv \mathrm{CH}\right)\right.$ $\left.{ }_{6}\left(\mathrm{H}_{2} \mathrm{O}\right)_{4}\right] \cdot 2 \mathrm{H}_{2} \mathrm{O}$ complex. Dalton Trans. 2007;(23):2450-6. 
21. Imaz I, Luis F, Carbonera C, Ruiz-Molina D, Maspoch D. Single-molecule magnet behaviour in metal-organic nanospheres generated by simple precipitation of $\mathrm{Mn}_{12} \mathrm{O}_{12}$ clusters. Chem Commun. 2008;(10):1202-4.

22. van Slageren J, Rosa P, Caneschi A, Sessoli R, Casellas H, Rakitin YV, et al. Static and dynamic magnetic properties of an [Fe13] cluster. Phys Rev B. 2006 Jan 24;73(1):014422. https://link.aps.org/doi/10.1103/PhysRevB.73.014422

23. Habib F, Long J, Lin P-H, Korobkov I, Ungur L, Wernsdorfer W, et al. Supramolecular architectures for controlling slow magnetic relaxation in field-induced single-molecule magnets. Chem Sci. 2012;3(6):2158.

24. Cosquer G, Pointillart F, Golhen S, Cador O, Ouahab L. Slow Magnetic Relaxation in Condensed versus Dispersed Dysprosium(III) Mononuclear Complexes. Chem Eur J. 2013 Jun 10;19(24):7895-903.

25. Huang G, Daiguebonne C, Calvez G, Suffren Y, Guillou O, Guizouarn T, et al. Strong Magnetic Coupling and Single-Molecule-Magnet Behavior in Lanthanide-TEMPO Radical Chains. Inorg Chem. 2018 Sep 4;57(17):11044-57.

26. Huang G, Calvez G, Suffren Y, Daiguebonne C, Freslon S, Guillou O, et al. Closing the Circle of the Lanthanide-Murexide Series: Single-Molecule Magnet Behavior and Near-Infrared Emission of the NdIII Derivative. Magnetochemistry. 2018 Oct 3;4(4):44.

27. Fort A, Rettori A, Villain J, Gatteschi D, Sessoli R. Mixed Quantum-Thermal Relaxation in Mn 12 Acetate Molecules. Phys Rev Lett. 1998 Jan 19;80(3):612-5.

28. Ding Y-S, Yu K-X, Reta D, Ortu F, Winpenny REP, Zheng Y-Z, et al. Field- and temperature-dependent quantum tunnelling of the magnetisation in a large barrier single-molecule magnet. Nat Commun. 2018 Dec;9(1):3134. http://www.nature.com/articles/s41467-018-05587-6

29. Orbach R. Spin-lattice relaxation in rare-earth salts. Proc R Soc Lond Ser Math Phys Sci. 1961 Dec 5;264(1319):458-84.

30. Van Vleck JH. Paramagnetic Relaxation Times for Titanium and Chrome Alum. Phys Rev. 1940 Mar 1;57(5):426-47.

31. Stoll S, Schweiger A. EasySpin, a comprehensive software package for spectral simulation and analysis in EPR. J Magn Reson. 2006 Jan;178(1):42-55.

32. Chilton NF, Anderson RP, Turner LD, Soncini A, Murray KS. PHI: A powerful new program for the analysis of anisotropic monomeric and exchange-coupled polynuclear $d$ - and $f$-block complexes. J Comput Chem. 2013 May 15;34(13):1164-75.

33. Borrás-Almenar JJ, Clemente-Juan JM, Coronado E, Tsukerblat BS. MAGPACK $^{1}$ A package to calculate the energy levels, bulk magnetic properties, and inelastic neutron scattering spectra of high nuclearity spin clusters: Software News and Updates. J Comput Chem. 2001 Jul 15;22(9):985-91.

34. Cole KS, Cole RH. Dispersion and Absorption in Dielectrics I. Alternating Current Characteristics. J Chem Phys. 1941 Apr;9(4):341-51.

35. Davidson DW, Cole RH. Dielectric Relaxation in Glycerol, Propylene Glycol, and $n$-Propanol. J Chem Phys. 1951 Dec;19(12):1484-90. 
36. Havriliak S, Negami S. A complex plane analysis of a-dispersions in some polymer systems. J Polym Sci Part C Polym Symp. 1966;14(1):99-117.

37. Klitgaard SK, Galsbøl F, Weihe H. Angular variation of linewidths in single-crystal EPR spectra. Spectrochim Acta A Mol Biomol Spectrosc. 2006 Mar;63(4):836-9.

38. Boudalis AK, Rogez G, Turek P. Determination of the Distributions of the Spin-Hamiltonian Parameters in Spin Triangles: A Combined Magnetic Susceptometry and Electron Paramagnetic Resonance Spectroscopic Study of the Highly Symmetric $\left[\mathrm{Cr}_{3} \mathrm{O}(\mathrm{PhCOO})_{6}(\mathrm{py})_{3}\right]\left(\mathrm{ClO}_{4}\right) \cdot 0.5 \mathrm{py}$. Inorg Chem. 2018 Nov 5;57(21):13259-69.

39. Clayton JA, Keller K, Qi M, Wegner J, Koch V, Hintz H, et al. Quantitative analysis of zero-field splitting parameter distributions in Gd(III) complexes. Phys Chem Chem Phys. 2018;20(15):10470-92.

40. Ishikawa N, Sugita M, Ishikawa T, Koshihara S, Kaizu Y. Lanthanide Double-Decker Complexes Functioning as Magnets at the Single-Molecular Level. J Am Chem Soc. 2003 Jul;125(29):8694-5.

41. Accorsi S, Barra A-L, Caneschi A, Chastanet G, Cornia A, Fabretti AC, et al. Tuning Anisotropy Barriers in a Family of Tetrairon(III) Single-Molecule Magnets with an $S=5$ Ground State. J Am Chem Soc. 2006 Apr;128(14):4742-55.

42. Papatriantafyllopoulou C, Wernsdorfer W, Abboud KA, Christou G. Mn 21 Dy Cluster with a Record Magnetization Reversal Barrier for a Mixed 3d/4f Single-Molecule Magnet. Inorg Chem. 2011 Jan 17;50(2):421-3.

43. Le Roy JJ, Ungur L, Korobkov I, Chibotaru LF, Murugesu M. Coupling Strategies to Enhance Single-Molecule Magnet Properties of Erbium-Cyclooctatetraenyl Complexes. J Am Chem Soc. 2014 Jun 4;136(22):8003-10.

44. Liu J, Chen Y-C, Liu J-L, Vieru V, Ungur L, Jia J-H, et al. A Stable Pentagonal Bipyramidal Dy(III) Single-Ion Magnet with a Record Magnetization Reversal Barrier over 1000 K. J Am Chem Soc. 2016 Apr 27;138(16):5441-50.

45. Liu J-L, Wu J-Y, Chen Y-C, Mereacre V, Powell AK, Ungur L, et al. A Heterometallic Fe ${ }^{\mathrm{II}}$-Dy ${ }^{\mathrm{III}}$ Single-Molecule Magnet with a Record Anisotropy Barrier. Angew Chem Int Ed. 2014 Nov 17;53(47):12966-70.

46. Upadhyay A, Singh SK, Das C, Mondol R, Langley SK, Murray KS, et al. Enhancing the effective energy barrier of a Dy(III) SMM using a bridged diamagnetic Zn(II) ion. Chem Commun. 2014;50(64):8838-41.

47. Goodwin CAP, Ortu F, Reta D, Chilton NF, Mills DP. Molecular magnetic hysteresis at 60 kelvin in dysprosocenium. Nature. 2017 Aug;548(7668):439-42.

48. Guo F-S, Day BM, Chen Y-C, Tong M-L, Mansikkamäki A, Layfield RA. Magnetic hysteresis up to 80 kelvin in a dysprosium metallocene single-molecule magnet. Science. 2018 Dec 21;362(6421):1400-3.

49. Reta D, Chilton NF. Uncertainty estimates for magnetic relaxation times and magnetic relaxation parameters. Phys Chem Chem Phys. 2019;21(42):23567-75. 
50. Liu J-L, Chen Y-C, Tong M-L. Symmetry strategies for high performance lanthanide-based single-molecule magnets. Chem Soc Rev. 2018;47(7):2431-53.

51. Chen W-B, Chen Y-C, Huang G-Z, Liu J-L, Jia J-H, Tong M-L. Cyclic OFF/Part/ON switching of single-molecule magnet behaviours via multistep single-crystal-to-single-crystal transformation between discrete Fe(II)-Dy(III) complexes. Chem Commun. 2018;54(77):10886-9.

52. Boudalis AK, Sanakis Y, Clemente-Juan JM, Donnadieu B, Nastopoulos V, Mari A, et al. A Family of Enneanuclear Iron(II) Single-Molecule Magnets. Chem Eur J. 2008 Mar 7;14(8):2514-26.

53. Boudalis AK, Donnadieu B, Nastopoulos V, Clemente-Juan JM, Mari A, Sanakis Y, et al. A Nonanuclear Iron(II) Single-Molecule Magnet. Angew Chem Int Ed. 2004 Apr 19;43(17):2266-70.

54. Horii Y, Kishiue S, Damjanović M, Katoh K, Breedlove BK, Enders M, et al. Supramolecular Approach for Enhancing Single-Molecule Magnet Properties of Terbium(III)-Phthalocyaninato Double-Decker Complexes with Crown Moieties. Chem Eur J. 2018 Mar 20;24(17):4320-7.

55. Chakarawet K, Bunting PC, Long JR. Large Anisotropy Barrier in a Tetranuclear Single-Molecule Magnet Featuring Low-Coordinate Cobalt Centers. J Am Chem Soc. 2018 Feb 14;140(6):2058-61.

56. Feng X, Hwang SJ, Liu J-L, Chen Y-C, Tong M-L, Nocera DG. Slow Magnetic Relaxation in Intermediate Spin $S=3 / 2$ Mononuclear Fe(III) Complexes. J Am Chem Soc. 2017 Nov 22;139(46):16474-7.

57. Bhowmick I, Roehl AJ, Neilson JR, Rappé AK, Shores MP. Slow magnetic relaxation in octahedral low-spin Ni(III) complexes. Chem Sci. 2018;9(31):6564-71.

58. Moreno Pineda E, Chilton NF, Marx R, Dörfel M, Sells DO, Neugebauer P, et al. Direct measurement of dysprosium(III) ${ }^{\cdots}$ dysprosium(III) interactions in a single-molecule magnet. Nat Commun. 2014 Dec;5(1):5243. http://www.nature.com/articles/ncomms6243

59. Escalera-Moreno L, Baldoví JJ, Gaita-Ariño A, Coronado E. Spin states, vibrations and spin relaxation in molecular nanomagnets and spin qubits: a critical perspective. Chem Sci. 2018;9(13):3265-75.

60. Wäckerlin C, Donati F, Singha A, Baltic R, Rusponi S, Diller K, et al. Giant Hysteresis of Single-Molecule Magnets Adsorbed on a Nonmagnetic Insulator. Adv Mater. 2016 Jul;28(26):5195-9.

How to cite this article:

Boudalis AK. SMM-ET: An SMM Evaluation Tool for the Quantitative Treatment of Ac Susceptibility and Magnetic Hysteresis Data. Quantum Mater Res. 2020;1:e200004. https://doi.org/10.20900/qmr20200004 